\title{
Epsin deficiency promotes lymphangiogenesis through regulation of VEGFR3 degradation in diabetes
}

\author{
Hao Wu, ${ }^{1}$ H.N. Ashiqur Rahman, ${ }^{1}$ Yunzhou Dong, ${ }^{1}$ Xiaolei Liu, ${ }^{2}$ Yang Lee, ${ }^{1}$ Aiyun Wen, ${ }^{1}$ Kim H.T. To, ${ }^{3}$ Li Xiao, ${ }^{4}$ Amy E. Birsner, ${ }^{1}$ \\ Lauren Bazinet, ${ }^{1}$ Scott Wong, ${ }^{1}$ Kai Song, ${ }^{1}$ Megan L. Brophy, ${ }^{1,5}$ M. Riaj Mahamud, ${ }^{6}$ Baojun Chang, ${ }^{7}$ Xiaofeng Cai, ${ }^{6}$ Satish Pasula, ${ }^{6}$ \\ Sukyoung Kwak, ${ }^{1}$ Wenxia Yang, ${ }^{4}$ Joyce Bischoff, ${ }^{1}$ Jian Xu, ${ }^{8}$ Diane R. Bielenberg, ${ }^{1}$ J. Brandon Dixon, ${ }^{3}$ Robert J. D’Amato, \\ R. Sathish Srinivasan, ${ }^{6}$ and Hong Chen ${ }^{1}$ \\ 'Vascular Biology Program, Harvard Medical School, Boston Children's Hospital, Boston, Massachusetts, USA. ²enter for Vascular and Developmental Biology, Northwestern University Feinberg School of \\ Medicine, Chicago, Illinois, USA. 3Parker H. Petit Institute for Bioengineering and Bioscience, Georgia Institute of Technology, Atlanta, Ceorgia, USA. ${ }^{4}$ Department of Nephrology, the Second Xiangya Hospital, \\ Central South University, Changsha, Hunan, China. ${ }^{5}$ Department of Biochemistry and Molecular Biology, University of Oklahoma Health Science Center, Oklahoma City, Oklahoma, USA. ${ }^{6}$ Cardiovascular \\ Biology Program, Oklahoma Medical Research Foundation, Oklahoma City, Oklahoma, USA. 'Vascular Medicine Institute, Pulmonary, Allergy and Critical Care Division, University of Pittsburgh School of \\ Medicine, Pittsburgh, Pennsylvania, USA. ${ }^{8}$ Department of Medicine, University of Oklahoma Health Science Center, Oklahoma City, Oklahoma, USA.
}

\begin{abstract}
Impaired lymphangiogenesis is a complication of chronic complex diseases, including diabetes. VEGF-C/VEGFR3 signaling promotes lymphangiogenesis, but how this pathway is affected in diabetes remains poorly understood. We previously demonstrated that loss of epsins 1 and 2 in lymphatic endothelial cells (LECs) prevented VEGF-Cinduced VEGFR3 from endocytosis and degradation. Here, we report that diabetes attenuated VEGF-C-induced lymphangiogenesis in corneal micropocket and Matrigel plug assays in WT mice but not in mice with inducible lymphatic-specific deficiency of epsins 1 and 2 (LEC-iDKO). Consistently, LECs isolated from diabetic LEC-iDKO mice elevated in vitro proliferation, migration, and tube formation in response to VEGF-C over diabetic WT mice. Mechanistically, ROS produced in diabetes induced c-Src-dependent but VEGF-C-independent VEGFR3 phosphorylation, and upregulated epsins through the activation of transcription factor AP-1. Augmented epsins bound to and promoted degradation of newly synthesized VECFR3 in the Golgi, resulting in reduced availability of VEGFR3 at the cell surface. Preclinically, the loss of lymphatic-specific epsins alleviated insufficient lymphangiogenesis and accelerated the resolution of tail edema in diabetic mice. Collectively, our studies indicate that inhibiting expression of epsins in diabetes protects VEGFR3 against degradation and ameliorates diabetes-triggered inhibition of lymphangiogenesis, thereby providing a novel potential therapeutic strategy to treat diabetic complications.
\end{abstract}

\section{Introduction}

The lymphatic vascular system is composed of draining lymphatic vessels, lymph nodes, and lymphoid organs. Lymphatic vessels are blind-ended, unidirectional, absorptive vessels. They play critical roles in tissue fluid homeostasis, immune cell trafficking, and lipid absorption (1-3). Primary defects in lymphatic growth, or secondary conditions such as filarial infection or surgical removal of lymph nodes, will cause impaired lymphatic drainage. Loss of lymphatic function will result in edema, malabsorption syndromes, and immune defects that may be fatal (1-3). Given the importance of lymphatics in maintaining fluid balance and mediating the inflammatory response in the skin, it is perhaps not surprising that lymphatic dysfunction has been implicated in diabetes (4-8). Diabetes is clinically characterized by hyperglycemia due to insufficiency of insulin in the body either through lack of insulin

Authorship note: HW, HNAR, and YD contributed equally to this work. Conflict of interest: The authors have declared that no conflict of interest exists. Submitted: July 5, 2017; Accepted: June 26, 2018.

Reference information: J Clin Invest. 2018;128(9):4025-4043.

https://doi.org/10.1172/JC196063. production or an impaired effectiveness of insulin to lower blood sugar, primarily due to a loss of insulin sensitivity (9). In diabetes mellitus, hyperglycemia-induced oxidative stress produced from mitochondria is the earliest event in the development of cardiovascular complications (10). Impaired wound healing is a common complication in diabetes, which also manifests in secondary lymphedema (11-13), suggesting that the lymphatic system plays a functional role in mitigating diabetic complications. Moreover, functional lymphangiogenesis, along with its counterpart angiogenesis, is required to accelerate wound healing in both normal and diabetic conditions $(4,14)$. However, normal function of the lymphatic system is commonly interrupted in diabetes, as recent studies show that nitric oxide signaling is attenuated in type 2 diabetes, disrupting lymphatic vascular integrity (7).

Lymphangiogenic growth factors such as VEGF-C and VEGF$\mathrm{D}$ are therapeutic targets for enhancing lymphatic vessel growth through their action on VEGFR3 receptors (15-17). VEGFR3 is present in all endothelial cells during the early postnatal development period. However, in mature adult vessels VEGFR3 becomes restricted to the lymphatic endothelium (18-21). The VEGF-C/ VEGFR3 signaling axis is the most well-studied central pathway 
A

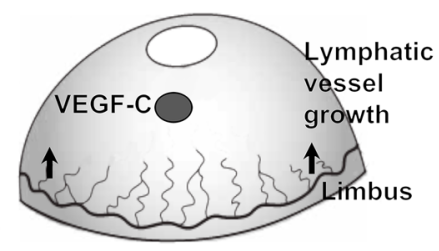

C

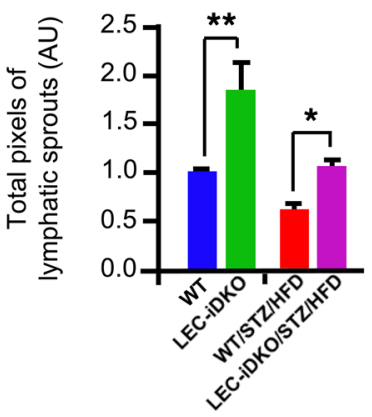

B

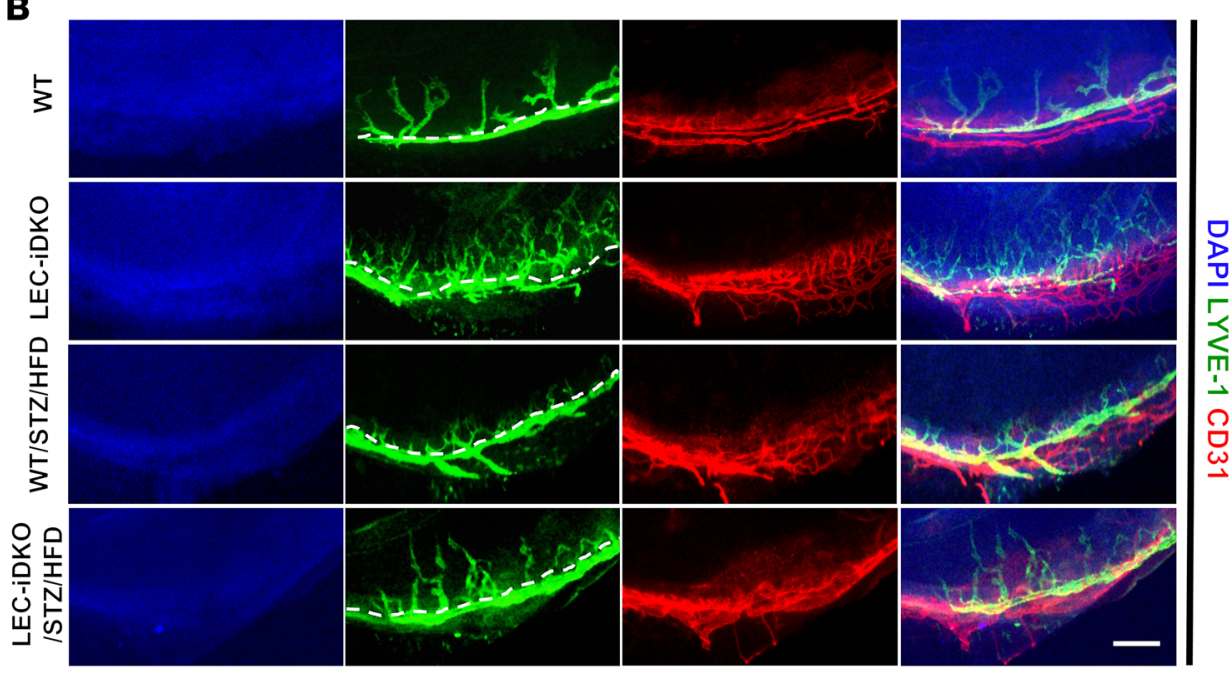

D

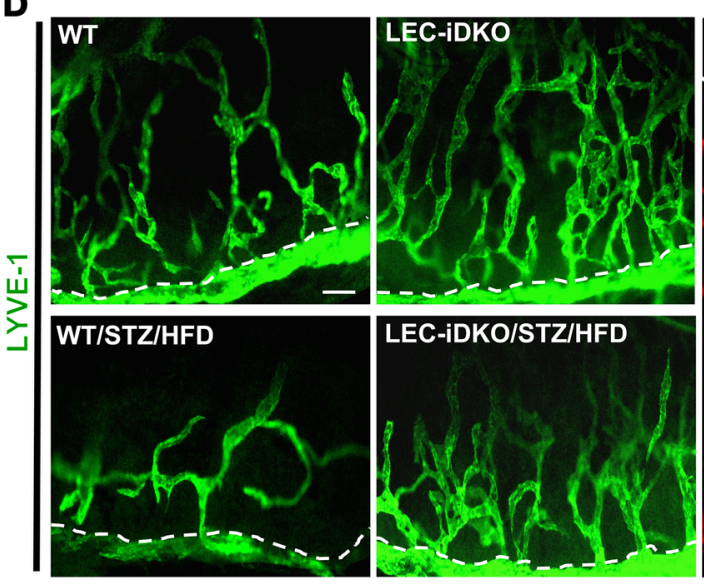

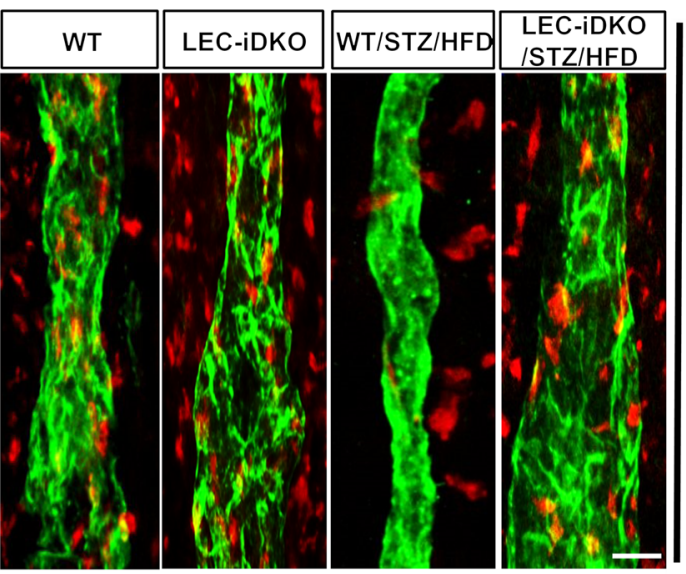

G

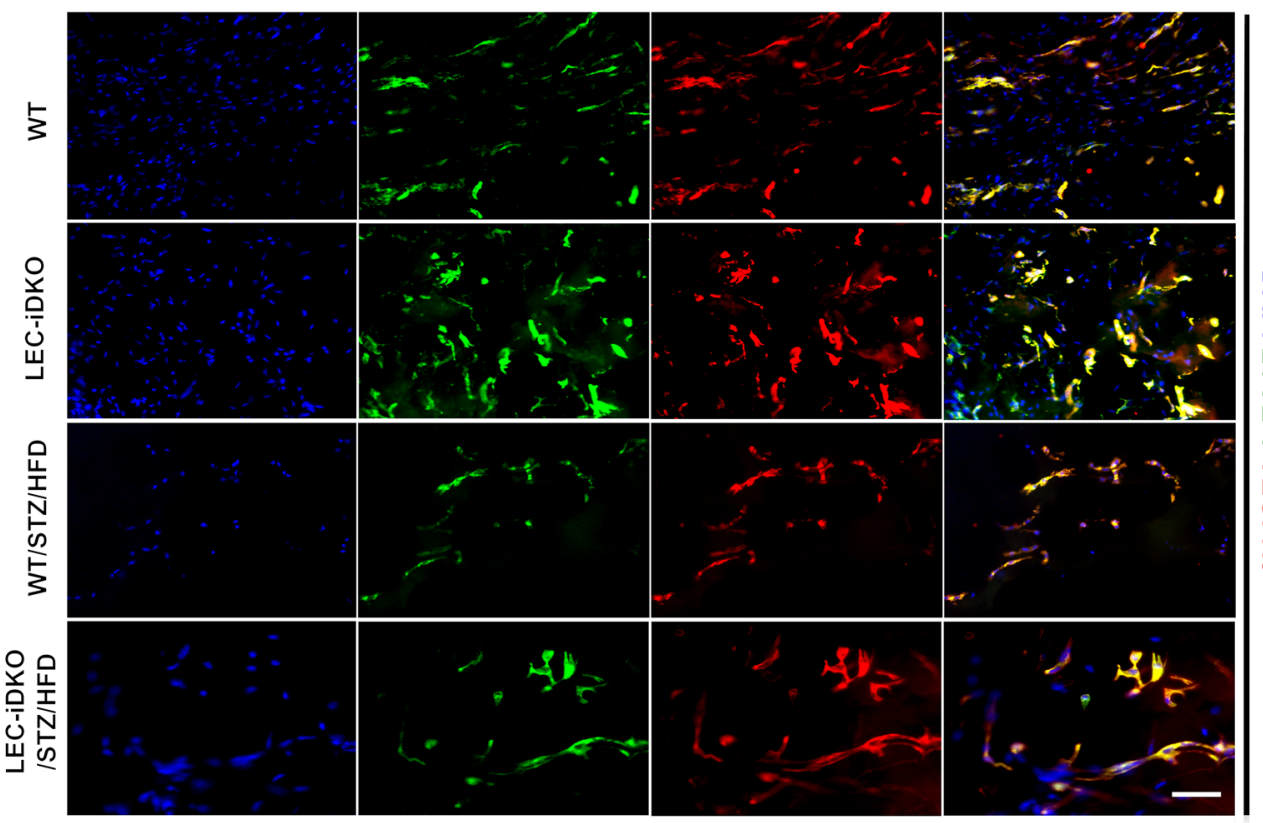

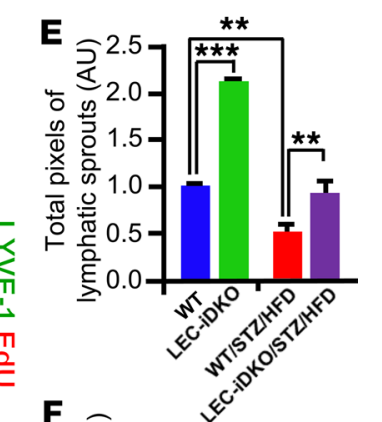

F

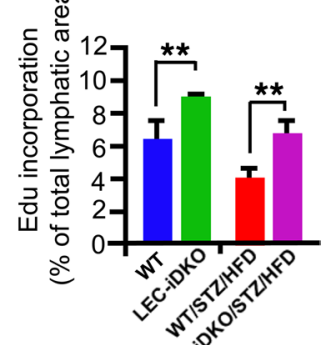

H
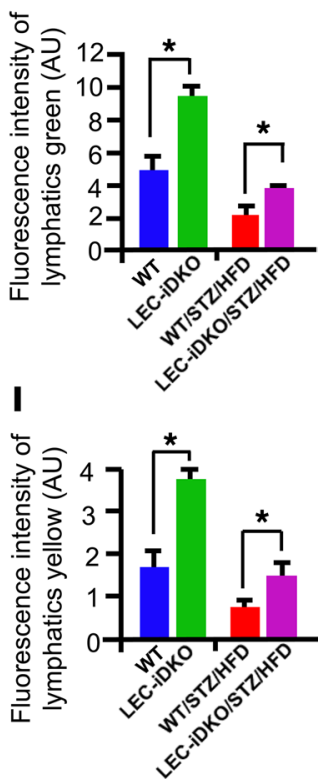
Figure 1. Lymphatic endothelial cell-specific deficiency of epsins promotes lymphangiogenesis in vivo. (A) A schematic diagram of corneal micropocket for pellet implantation. Release of the lymphangiogenic factor VEGF-C from the pellet induces lymphatic vessel growth in the limbus. (B and C) Whole-mount immunofluorescence staining in corneal tissue using antibodies for DAPI (blue), LYVE-1 (green), and CD31 (red) at day 7 after VEGF-C implantation. Quantification of lymphatic vessel growth area in B. (D-F) High-magnification images ( $\times 10$, left panel of $\mathbf{D}$ ) show substantial lymphatic plexus in LEC-iDKO mice. Quantification of lymphatic vessel growth in E. Representative confocal Z-stack images show the lymphatic vessels stained with LYVE-1 (green) and Edu (red) in the right panel of $\mathbf{D}$. Quantification of Edu incorporation in lymphatic vessels in F. (G-I) Lymphangiogenesis in Matrigel plug assay. Lymphatic vessels were stained with LYVE-1 (green) and VEGFR3 (red). Quantifications of neovascularization of lymphatic vessels and VEGFR3 localized in the neovascularized lymphatic vessels (yellow) are shown in $\mathbf{H}$ and $\mathbf{I}$. Data are mean $\pm \mathrm{SEM}$, $n=5$. ${ }^{*} P<0.05$; ${ }^{* *} P<0.01$; ${ }^{* *} P<0.001$, by 2 -way ANOVA followed by Tukey's post hoc test. Scale bars: $200 \mu \mathrm{m}$ (B), $100 \mu \mathrm{m}$ (D, left panel), 20 $\mu \mathrm{m}$ (D, right panel), $50 \mu \mathrm{m}$ (C).

for lymphangiogenesis $(2,3,20,22-24)$. VEGF-C-induced dimerization and autophosphorylation of VEGFR3 activates several downstream signaling events, including Akt and ERK signaling pathways that drive lymphangiogenesis (25-27). Similar to human diabetic patients, wound healing in genetically diabetic $(d b / d b)$ mice is impaired; however, exogenous VEGF-C administration can markedly improve wound healing in these mice (5). Given that elevated VEGF-C has therapeutic implications, at least in preclinical murine diabetic models, we reason that enhanced cell-surface expression of VEGFR3 would similarly offer beneficial effects of increased ligand concentration as a means of alleviating diabetic complications. However, cell-surface accumulation of VEGF receptors is crippled in diabetes as demonstrated by a recent study showing that high glucose levels reduce newly synthesized VEGFR2 trafficking from the Golgi compartment to the cell surface (28). Whether and how VEGFR3 cell-surface expression is similarly downregulated in diabetes is unclear but it is a highly medically relevant question. The precise molecular mechanism regulating VEGFR3-mediated lymphangiogenesis and other possible regulators under diabetic conditions remains largely unknown.

Epsins are a family of highly conserved multifunctional endocytic adaptor proteins that mediate clathrin-dependent endocytosis of a subset of ubiquitinated cell-surface proteins (29-31). Epsins 1 and 2 are ubiquitously expressed in most tissues where they perform overlapping functions $(30,32,33)$. Synonymous with their roles, epsins 1 and 2 facilitate the internalization and downregulation of VEGFR2 in vascular endothelial cells and VEGFR3 in lymphatic endothelial cells (LECs) $(34,35)$. We previously demonstrated that VEGFR3 signaling is modulated by endocytosis and that epsins play an important role in temporal and spatial control of VEGFR3 signaling. Further, previous studies have established the inverse correlation between epsin levels and VEGFR3 signaling in the context of collecting lymphatic vessel maturation (34). It is yet unknown whether inducible deletion of epsins in adult lymphatic endothelium similarly upregulates VEGFR3 signaling and whether this enhanced signaling could be manipulated to enhance reparative lymphangiogenesis in pathological conditions such as diabetes. The dynamic role of VEGFR3 turnover during lymphangiogenesis, coupled with our discovery of epsins' effect on the regulation of VEGFR3 expression on the cell surface, strongly suggests the involvement of epsins in impaired lymphangiogenesis in diabetes.

In this study, we investigated the role of epsins in VEGFR3dependent lymphangiogenesis under diabetic conditions by generating an adult inducible mouse model of lymphatic endothelial cell-specific epsin deletion driven by Prox1-Cre-ER ${ }^{T 2}$ with the administration of 4-hydroxytamoxifen. To mimic type 2 diabetes, these 8- to 10-week-old adult mice were treated with streptozotocin (STZ) and fed a high-fat diet (HFD) in order to maintain hyperglycemia (referred to herein as LEC-iDKO/STZ/HFD mice). Many animal models have been created to investigate the mechanisms governing lymphatic development and the diseases associated with lymphatic dysfunction and abnormal lymphangiogenesis, including diabetes (11, 36-40). For example, models within the cornea have been widely used to investigate de novo lymphangiogenesis. The cornea is an advantageous location for such studies because of the corneal avascularity (41-43). Using the corneal micropocket assay, VEGF-C was confirmed to be a potent lymphangiogenic factor and to induce corneal lymphangiogenesis through the activation of VEGFR3 $(44,45)$. We used mouse corneal micropocket and Matrigel plug assays, mouse tail lymphedema models, and in vitro assays to investigate the roles of epsins in lymphangiogenesis under diabetic conditions. Our data demonstrated that depletion of epsins promotes lymphangiogenesis in diabetes by modulating VEGFR3 expression levels and signaling.

\section{Results}

Generation of WT/STZ/HFD and LEC-iDKO/STZ/HFD diabetic mice displaying type 2 diabetes metabolic syndrome. To circumvent developmental defects intrinsic to mice with constitutive lymphatic epsin deficiency driven by LYVE-1-Cre, we created an inducible lymphatic endothelial cell-specific epsin deletion driven by the Prox1-Cre-ER $R^{T 2}$ mouse model by crossing Epn $1^{t / f l} / E p n 2^{-/-}$mice with Prox1-Cre-ER $R^{T 2}$ mice (Supplemental Table 1; supplemental material available online with this article; https://oi.org/10.1172/JCI96063DS1). These adult Epn1 $1^{f / f l} / E p n 2^{-/} /$Prox1-Cre-ER ${ }^{T 2}$ mice (LECs on epsin 2-null background) were selectively ablated of epsin 1 in LECs by administering 4-hydroxytamoxifen at 8 to 10 weeks of age. Epn $1^{f / f t} / E p n 2^{-1-}$ littermate mice without Prox1-Cre-ER ${ }^{T 2}$ or WT mice with Prox1-Cre$E R^{T 2}$ were utilized as controls and are denoted as WT.

After verifying the successful deletion of epsins 1 and 2 in LECs by Western blot, lymphatic pump function was assessed in both WT and epsin-deficient (LEC-iDKO) mice using a near-infrared (NIR) imaging system to determine whether the LEC-iDKO mice showed any dysfunction in collecting lymphatic vessels. The NIR measurement in adult LEC-iDKO mice showed typical characteristics of packet transport and frequency indistinguishable from WT controls, suggesting loss of LEC epsins in adult mice has no effect on lymphatic pump function (Supplemental Figure 1, A-D). The LEC-iDKO mice have a similar collecting lymphatic phenotype and valve formation as the WT mice.

It has been shown that mice administered streptozotocin (STZ) and fed a high-fat diet (HFD) display symptoms reminiscent of type 2 diabetes (46-48). Both WT and LEC-iDKO mice were administrated a low dose of STZ ( $50 \mathrm{mg} / \mathrm{kg}$, intravenously), became hyperglycemic after 5 days of treatment, and were placed 
A

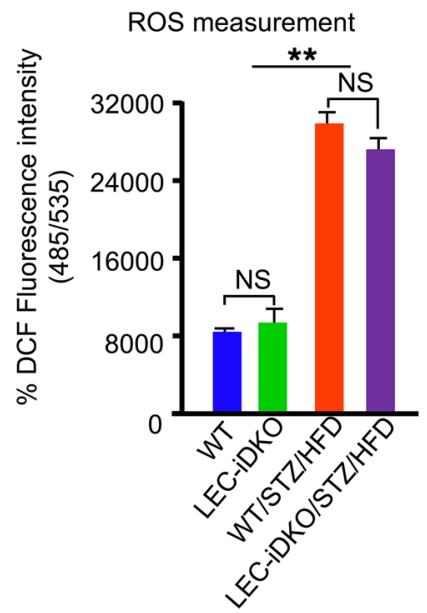

C

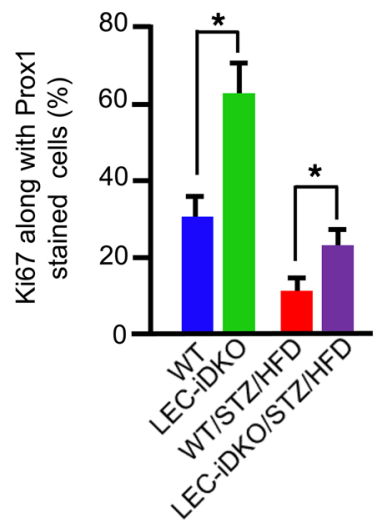

B
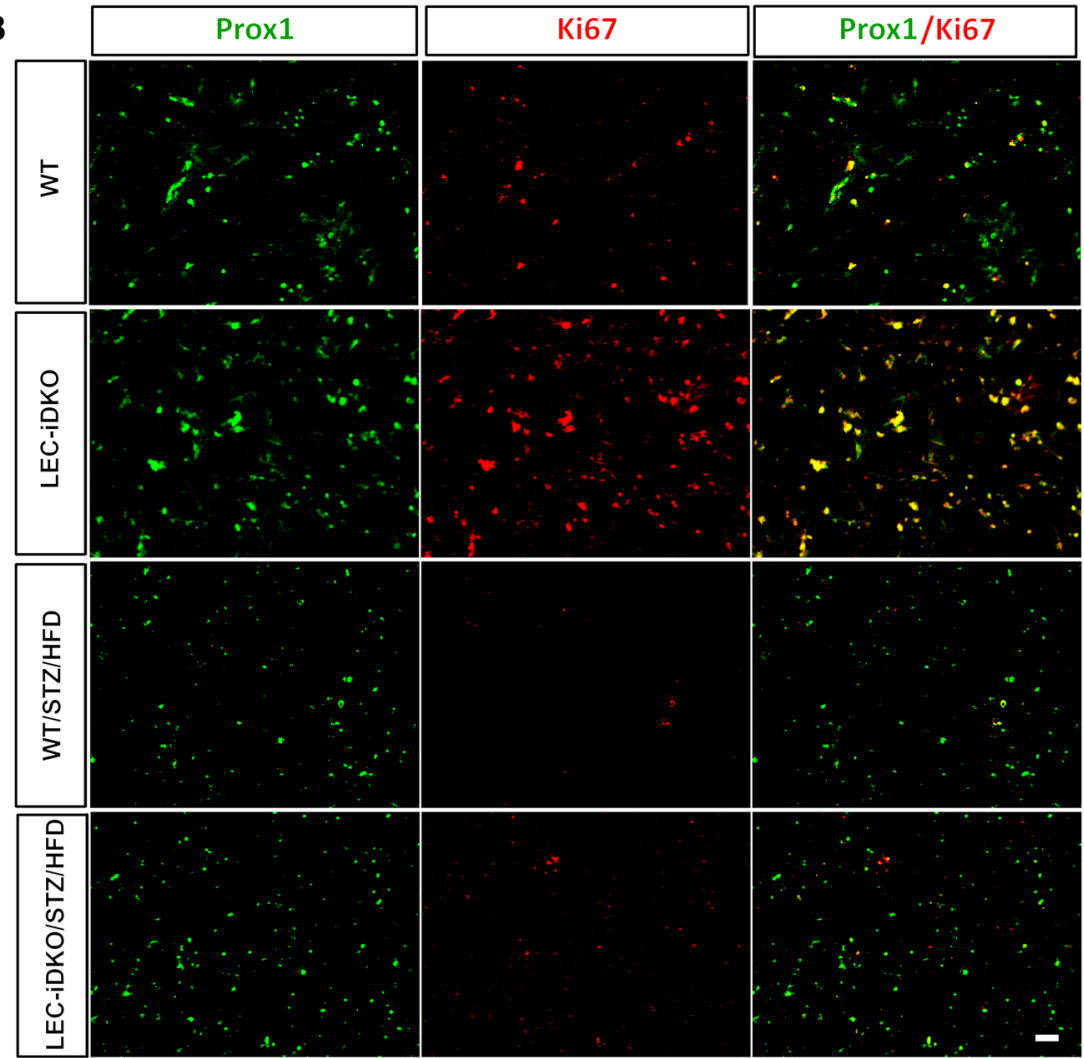

D

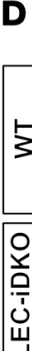

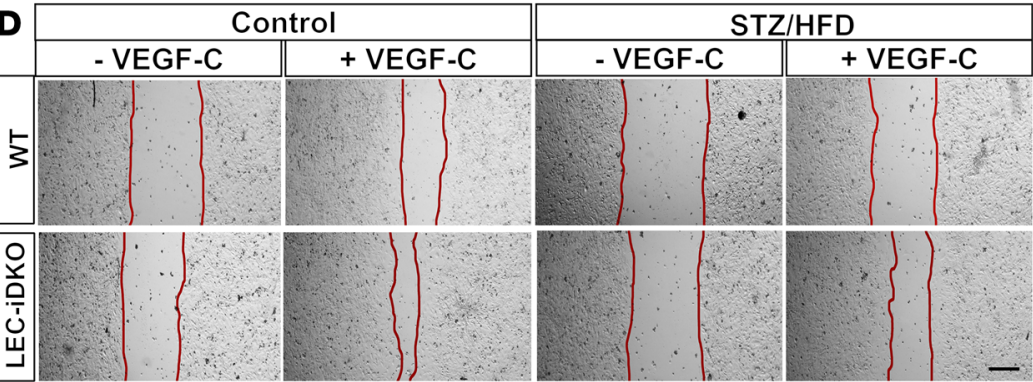

E WT LEC-IDKO

WT+VEGF-C LEC-iDKO+VEGF-C

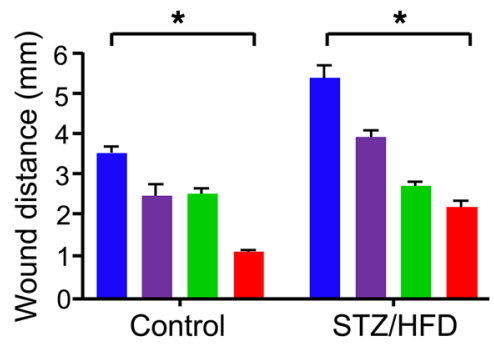

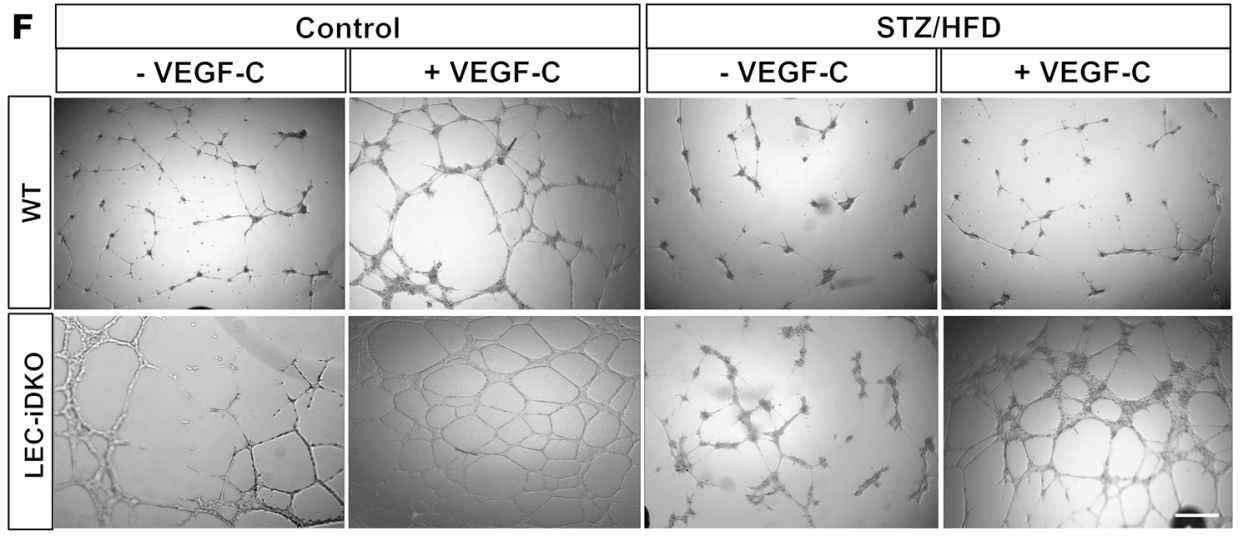

G

WT LEC-IDKO

LEC-iDKO+VEGF-C

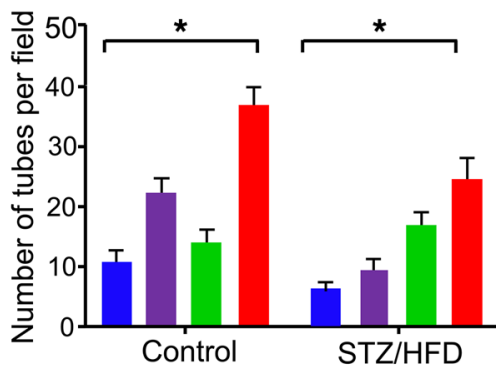


Figure 2. Epsin deficiency promotes proliferation, migration, and tube formation of lymphatic endothelial cells in vitro. (A) Fluorescence intensity of DCF indicates the ROS levels in primary LECs from WT, LEC-iDKO, WT/STZ/HFD, and LEC-iDKO/STZ/HFD mice. (B) Representative immunofluorescence staining of Ki67 (red) and Prox1 (green) in WT, LEC-iDKO, WT/ STZ/HFD, and LEC-iDKO/STZ/HFD LECs after treatment with $100 \mathrm{ng} / \mathrm{mL}$ VEGF-C for 24 hours. (C) Quantification of Ki67 staining in B. (D) Representative images of WT, LEC-iDKO, WT/STZ/HFD, and LEC-iDKO/STZ/HFD LECs cultured in LEC medium for 5 days, then subjected to a scratch assay in the absence or presence of $100 \mathrm{ng} / \mathrm{mL}$ VEGF-C for 12 hours. (E) Quantification of wound distance in $\mathbf{D}$. (F) Representative images of WT, LEC-iDKO, WT/STZ/HFD, and LEC-iDKO/STZ/HFD LECs cultured in LEC medium for 5 days, then subjected to a tube formation assay by culturing on Matrigel for 16 hours in the absence or presence of $100 \mathrm{ng} / \mathrm{mL}$ VEGF-C for 12 hours. (G) Quantification of tube formation in F. Data are mean \pm SEM, $n=7$. ${ }^{*} P<$ 0.05 , ${ }^{* *} P<0.01$, by 2-way ANOVA followed by Tukey's post hoc test. Scale bars: $100 \mu \mathrm{m}$ (B), $50 \mu \mathrm{m}$ (D and F).

on a HFD ( $60 \%$ of calories as fat) for 8 to 12 weeks (short term) to maintain hyperglycemia. These 2 groups of hyperglycemic type 2 diabetic mice were identified as WT/STZ/HFD and LEC-iDKO/ STZ/HFD. Glucose tolerance tests (GTTs), insulin tolerance tests (ITTs), and total cholesterol in both WT/STZ/HFDand LECiDKO/STZ/HFD mice displayed substantial symptoms of type 2 diabetes compared with normal control WT and LEC-iDKO mice (Supplemental Figure 2). Glucose disposal rate and whole-body insulin sensitivity in WT/STZ/HFD and LEC-iDKO/STZ/HFD mice were similar to each other, but were much worse than those in the control WT and LEC-iDKO mice, indicating that WT/STZ/ HFD and LEC-iDKO/STZ/HFD mice displayed typical hallmarks of type 2 diabetic syndromes. Furthermore, to verify that our STZ/ HFD diabetic models are different from the chronic inflammatory obesity models or other diabetic models with a long-term HFD feeding, we withheld the STZ treatment but fed the mice a HFD for 12 weeks and found that VEGFR3 levels in LECs did not change between WT and LEC-iDKO mice (data not shown).

Next, whole-mount immunofluorescence staining of mesenteric lymphatic vessels was performed using integrin $\alpha-9$ (red) and Prox1 (green) antibodies to highlight valve leaflets in the wholevalve regions. No significant difference in the number of Prox $1^{+}$ cells per leaflet, vessel diameter, maximum leaflet depth, and leaflet outer perimeter in lymphatic vessel valve leaflets was observed between WT, LEC-iDKO, WT/STZ/HFD, and LEC-iDKO/STZ/ HFD mice, suggesting that the structures of the lymphatic valves are not disrupted in STZ-induced diabetic mice (Supplemental Figure 1, $\mathrm{E}-\mathrm{I})$. All mice show both the normal collecting lymphatic vessel and normal lymphatic capillary phenotypes (Supplemental Figure 1).

Epsin deficiency promotes VEGF-C-stimulated lymphangiogenesis in diabetes in vivo. Taking advantage of the avascular characteristic of the mouse cornea, which offers a clean and unequivocal way to monitor newly formed vessels, we performed the mouse corneal micropocket assay to determine whether lymphaticspecific epsin deficiency promotes VEGF-C-induced lymphangiogenesis under diabetic conditions (45). Micropellets containing VEGF-C or vehicle control were implanted in micropockets in the eyes of WT, LEC-iDKO, WT/STZ/HFD, and LEC-iDKO/ STZ/HFD mice (Figure 1A). Corneal lymphangiogenic response was assessed on day 7 after implantation. Whole-mount immuno- fluorescence staining for LYVE-1 (green), CD31 (red), and DAPI (blue) of corneas demonstrated that LEC-iDKO mice displayed considerably increased lymphatic vessel growth as shown by elevated vessel density, branch points, and filopodia projections compared with those in WT mice when implanted with VEGF-Ccontaining micropellets (Figure 1, B and C). Notably, implantation of control micropellets that were lacking VEGF-C produced no lymphatic vessel growth and vessel branching as determined by staining of both LYVE-1 (green) and VEGFR3 (red) (Supplemental Figure 3). Importantly, nondiabetic WT mice exhibited increased lymphatic vascular density and elevated branching compared with diabetic WT/STZ/HFD mice, indicating that hyperglycemia impairs VEGF-C-induced lymphangiogenesis (Figure 1, B and D). However, this minimal VEGF-C-stimulated lymphangiogenesis was greatly enhanced in diabetic LEC-iDKO/STZ/HFD mice relative to diabetic WT/STZ/HFD mice (Figure 1, D and E), suggesting that epsins are responsible for the diminished lymphangiogenesis under diabetic conditions. Importantly, Z-stack images of Edu incorporation in LECs (Figure 1D, right panel; quantified in Figure $1 \mathrm{~F}$ ) revealed that LEC proliferation was suppressed in WT/STZ/HFD mice but increased in LEC-iDKO/STZ/HFD mice, suggesting that loss of epsins in LECs restores defective VEGF$\mathrm{C}$-induced lymphangiogenesis in diabetic mice (Supplemental Figure 4, yellow allows). Consistently, flow cytometric analysis of corneal LECs demonstrated a dramatic increase in the percentage of LYVE-1+ $1^{+}$corneal cells in LEC-iDKO/STZ/HFD mice compared with WT/STZ/HFD mice, suggesting that deficiency of epsins promotes VEGF-C-stimulated lymphangiogenesis in diabetes in vivo (Supplemental Figure 5).

To further investigate the potential of LEC-specific deficiency of epsins to ameliorate impaired VEGF-C-mediated lymphangiogenesis in diabetes, we employed another well-established in vivo lymphangiogenesis model, the Matrigel plug assay. Matrigel with or without VEGF-C was subcutaneously injected into mice, where it solidified to form plugs. On day 7 after injection, the Matrigel plugs were harvested and frozen sections of the plugs were stained for anti-LYVE-1 and VEGFR3 to visualize lymphatic vessel growth. As expected, VEGF-C stimulated more robust lymphatic vessel growth in LEC-iDKO mice compared with that in WT mice. Conversely, greatly reduced lymphatic vessel growth was observed in WT/STZ/HFD mice; however, this growth was substantially augmented in LEC-iDKO/STZ/HFD mice, increasing to an extent close to that seen in WT mice. The expression of VEGFR3 in lymphatic vessels corresponds with new growth in the lymphatic vascular plexus. VEGFR3 was more highly expressed in lymphatic vessels in LEC-iDKO mice compared with those in WT mice. Similarly, the expression level of VEGFR3 in LEC-iDKO/STZ/HFD mice was higher than that in WT/STZ/HFD mice (Figure 1, G-I). Furthermore, no macrophage infiltration was detected in Matrigel plugs of WT, LEC-iDKO, WT/STZ/HFD, and LEC-iDKO/STZ/ HFD mice, suggesting the VEGF-C-induced lymphangiogenesis in Matrigel plugs is not due to inflammatory neovascularization via macrophage recruitment (Supplemental Figure 6).

Collectively, our data indicate that epsin-mediated regulation potentially provides one of the major mechanisms underlying dysfunctional VEGF-C/VEGFR3-dependent in vivo lymphangiogenesis in hyperglycemic conditions. 
A Nondiabetics - Diabetic patients
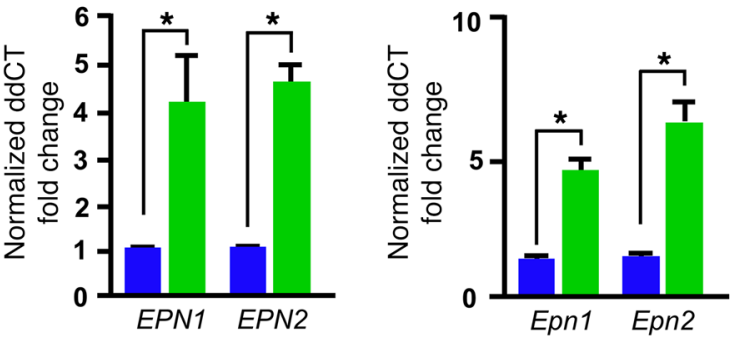

B

WT

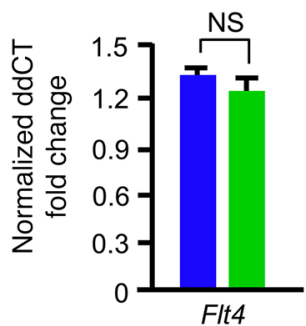

C

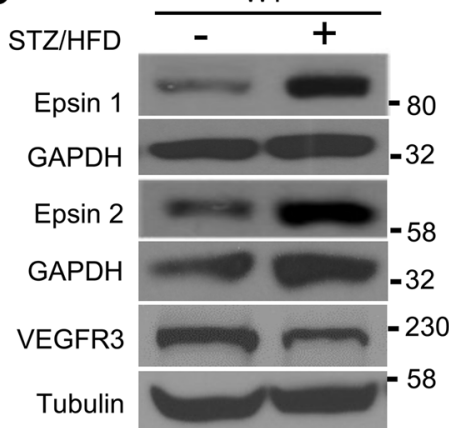

D

High glucose (Diabetic)

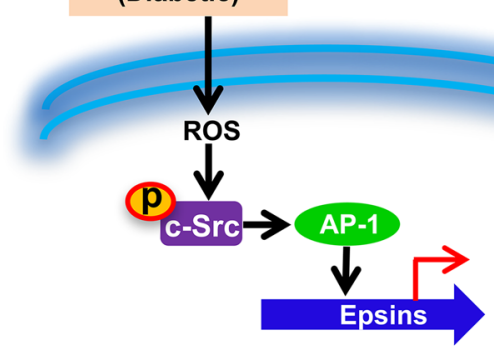

$F$

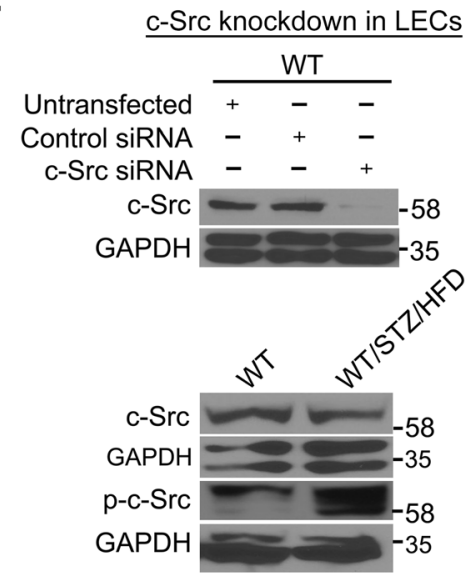

H

Luciferase assay - AP-1-blocking peptide

\section{- Control}

- AP-1:epsin promoter

AP-1:epsin promoter $+3 \mu \mathrm{M}$ peptide

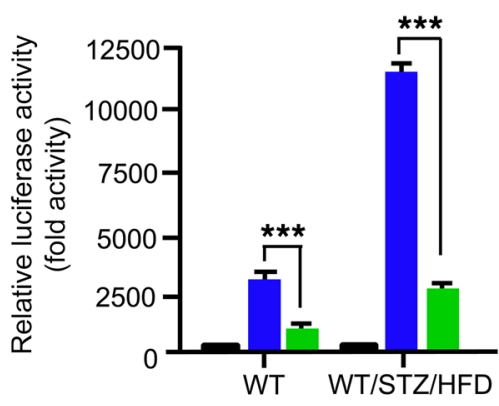

E

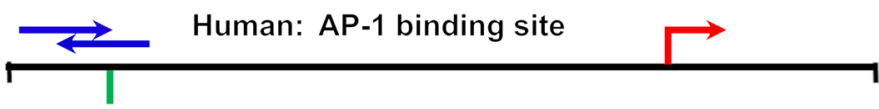

$-16.5 \mathrm{~KB}$

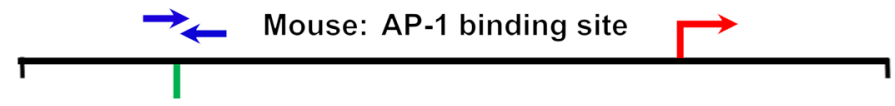

- 15.7KB $\longrightarrow$ Transcription Start Site of epsin 1

I AP-1 binding site $\longrightarrow$ ChIP-qPCR assay
G

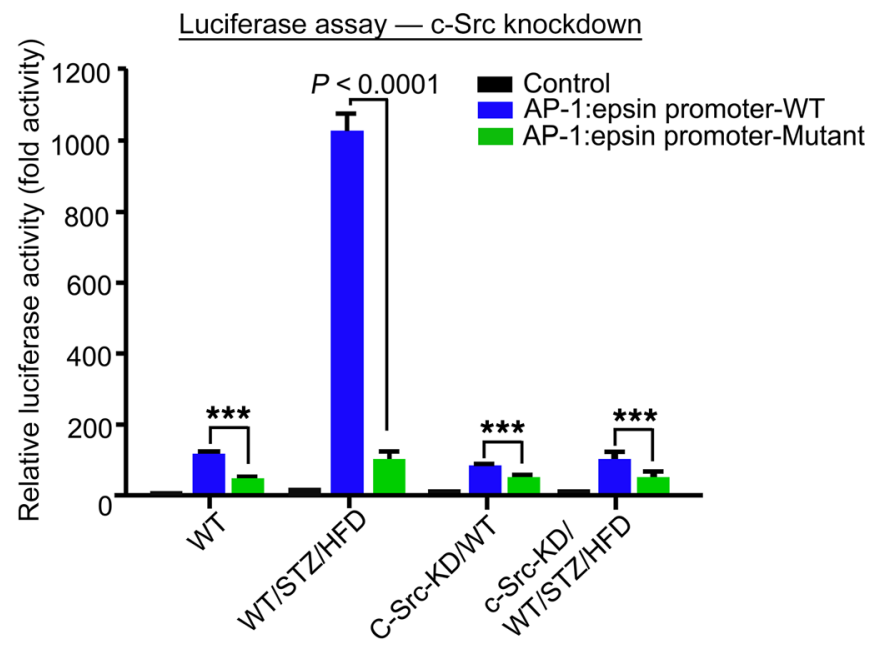


Figure 3. AP-1 drives upregulation of epsin expression via c-Src activation and VEGFR3 downregulation under diabetic conditions. (A) RT-qPCR for epsins 1 and 2 in LECs isolated from diabetic patients compared with nondiabetic persons (left panel) and in primary LECs from WT/STZ/HFD mice compared with WT (right panel). (B) RT-qPCR of VEGFR3 in both WT and LEC-iDKO primary LECs. (C) Western blot of epsins 1 and 2 in primary LECs from STZ-induced diabetic mice and normal WT mice. (D) Schematic diagram of the upregulation of epsins through c-Src under diabetic conditions. (E) Schematic diagram showing the transcription factor AP-1 binding sequence located at human and mouse epsin 1 promoter regions. (F) Western blot of WT mouse LECs with c-Src knockdown by c-Src siRNA (upper panel); c-Src and phosphorylated c-Src (p-c-Src) for levels in WT and WT/ STZ/HFD LEC cells (lower panel). (G) AP-1 expression vector was cotransfected with reporter plasmid pCL3-200 containing WT or mutant AP-1 binding sites into mouse LECs. (H) AP-1 expression vector was cotransfected with reporter plasmid pCL3-200 containing WT AP-1 binding sites into mouse LECs followed by treatment with an AP-1-blocking peptide. Blank vectors were used as the control. The luciferase activities of the WT and mutant reporter plasmids were measured and quantified. (I) ChIP assay to determine AP-1 binding to the epsin 1 promoter in LECs from STZ-induced diabetic mice and WT normal mice with and without c-Src knockdown (KD). Quantifications by using NIH ImageJ, $n=8$. Data are mean \pm SEM. ${ }^{*} P<0.05$; ${ }^{* *} P<0.001$, by 2-tailed Student's $t$ test (A and B), 1-way ANOVA followed by Tukey's post hoc test $(\mathbf{H})$, or 2-way ANOVA followed by Tukey's post hoc test ( $\mathbf{G}$ and $\mathbf{I})$.

Epsin deficiency promotes proliferation, migration, and network formation of lymphatic endothelial cells under diabetic conditions in vitro. To substantiate our in vivo findings, we examined the effect of epsin depletion on LEC proliferation, migration, and tube formation in vitro. We first examined whether LECs isolated from diabetic mice can, when cultured in cell growth medium containing high glucose similar to the concentration typical in diabetic patients, maintain the same oxidation stress caused by hyperglycemia in diabetes. The cellular ROS levels in LECs isolated from WT, LEC-iDKO, WT/STZ/HFD, and LEC-iDKO/STZ/HFD mice were assessed by measuring fluorescence of DCF ( $2^{\prime}, 7^{\prime}$-dichlorodihydrofluorescein). Fluorescence intensities of DCF indicated that ROS contents in LECs isolated from WT/STZ/HFD and LEC-iDKO/STZ/HFD diabetic mice were remarkably higher than those from WT and LEC-iDKO nondiabetic mice, suggesting that the freshly isolated LECs from diabetic mice maintain high oxidative stress in cell culture (Figure 2A). The proliferation capacity of these cells was assessed in the presence or absence of VEGF-C by immunostaining for Ki67, and migration capability was assessed using the scratch wound injury and Matrigel tube formation assays. In response to VEGF-C, LECs from LEC-iDKO mice showed increased proliferation (Figure 2, upper panel of $\mathrm{B}$ and $\mathrm{C}$ ), migration (Figure 2, left panels of D and E), and tube formation (Figure 2, the left panel of $\mathrm{F}$ and $\mathrm{G}$ ) compared with LECs from WT mice. Under diabetic conditions, proliferation (Figure 2B), migration (Figure 2D), and tube formation (Figure 2F) of LECs from diabetic WT/STZ/HFD mice were significantly decreased compared with those in nondiabetic WT mice. However, LECs from LEC-iDKO/ STZ/HFD mice showed dramatically enhanced VEGF-C-induced proliferation (Figure 2, B and C), migration (Figure 2, D and E), and tube formation (Figure 2, F and G) compared with LECs from WT/STZ/HFD mice. Consistent with in vivo observations, our data suggest that loss of epsins in LECs rescued VEGF-C-dependent lymphangiogenesis under diabetic conditions in vitro.
Epsins are upregulated in LECs of diabetic humans and mice, whereas VEGFR3 is downregulated in LECs of diabetic mice. To investigate whether the mechanism of failed lymphangiogenesis in the context of diabetes is epsin-dependent, and whether this epsin-dependent mechanism is of clinical relevance, we collected endothelial cells from clinically diabetic patients and checked the expression levels of epsins. Quantitative RT-PCR showed that EPNs 1 and 2 were significantly upregulated in diabetic patients compared with nondiabetic people, suggesting that an epsindependent mechanism is involved in impaired lymphangiogenesis in humans (Figure 3A, left panel). Furthermore, we found that a significant upregulation of Epns 1 and 2 in LECs from diabetic WT/ STZ/HFD mice compared with control WT mice (Figure 3A, right panel). However, mRNA levels of Flt4 in both WT and WT/STZ/ HFD mice were not statistically different (Figure 3B). The protein levels of epsins 1 and 2 in WT/STZ/HFD mice were markedly increased compared with those in WT control mice as demonstrated by Western blot (Figure 3C). In contrast, the total VEGFR3 level in WT/STZ/HFD mice was substantially decreased compared with that of the WT control mice, suggesting that in diabetic conditions, VEGFR3 is downregulated at the protein level but not at the transcription level. Consistently, epsins 1 and 2 were upregulated in LECs from $d b / d b$ mice at both the mRNA and protein levels, compared with those in LECs from WT mice. Importantly, there was no difference in mRNA levels of VEGFR3 between WT and $d b / d b$ mice but VEGFR3 was decreased at the protein level in $d b / d b$ mice (Supplemental Figure 7, A-C).

Taken together, our data demonstrate that epsins are upregulated in diabetes, whereas VEGFR3 is downregulated only at the protein level. Furthermore, this epsin-dependent mechanism of failed lymphangiogenesis in the context of diabetes is highly relevant in humans.

AP-1 drives upregulation of epsin expression via c-Src activation under diabetic conditions. As epsins are implicated in downregulating VEGF-C-dependent lymphangiogenesis under hyperglycemia, we reasoned that high levels of ROS may upregulate expression of epsins. ROS induces upregulation of transcription factors such as AP-1 and modulates signaling molecules, including VEGF (49). Interestingly, AP-1 transcriptional activity is increased in response to oxidative stress-induced c-Src activation (50). Bioinformatics analysis revealed a highly conserved, putative transcription factor AP-1 binding sequence, GCTGACCCAG, located $-16.5 \mathrm{~kb}$ upstream of human EPN1 transcription start site (TSS) and -15.7 kb upstream of mouse Epn1 TSS (Figure 3, D and E; Supplemental Figure 7E; Supplemental Table 2). AP-1 has potential binding sites located at the human and mouse epsin 2 promoter regions (Supplemental Table 3). This suggests that under diabetic conditions, AP-1 can drive the upregulation of both epsin 1 and 2 expression. Thus, we seek to determine whether diabetes drives c-Src- and AP-1-mediated upregulation of epsin expression (Figure 3, A and C). To evaluate whether AP- 1 is activated via c-Src activation under diabetic conditions, we first examined c-Src activity by assessing its phosphorylation status under diabetic conditions. We found that phosphorylation of c-Src was increased in LECs isolated from WT/STZ/HFD mice compared with those from nondiabetic WT mice (Figure 3F, lower panel); however, c-Src siRNA dramatically reduced c-Src expression and phosphorylation of c-Src (Figure 3F, 
A
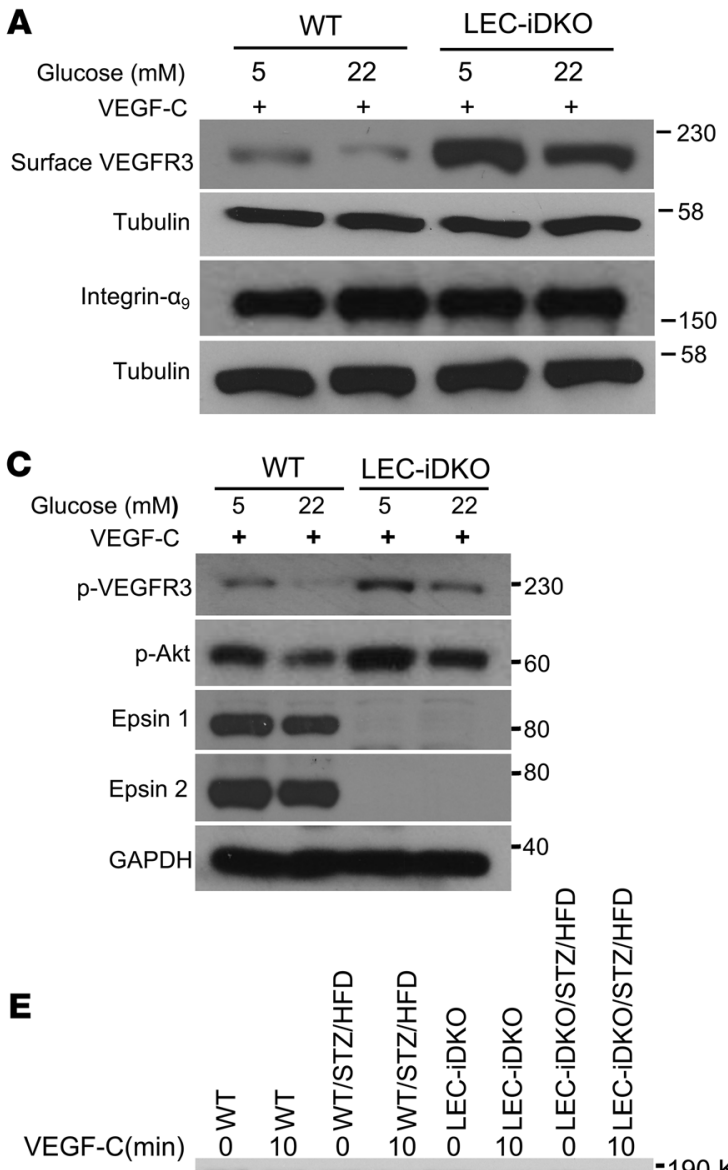

VEGFR $3==-\div=10$ PVEGFR $3 \equiv$ PAKT -. - - $-58 \mathrm{KDa}$ AKT $=-58 \mathrm{KDa}$ $\mathrm{pERK}=-46 \mathrm{KDa}$ $\mathrm{ERK}=-2-46 \mathrm{KDa}$ Epsin 1 - $-100 \mathrm{KDa}$ Epsin $1-\infty-100 \mathrm{KDa}$
Epsin $2--\infty-80 \mathrm{KDa}$ Tubulin $=-58 \mathrm{KDa}$

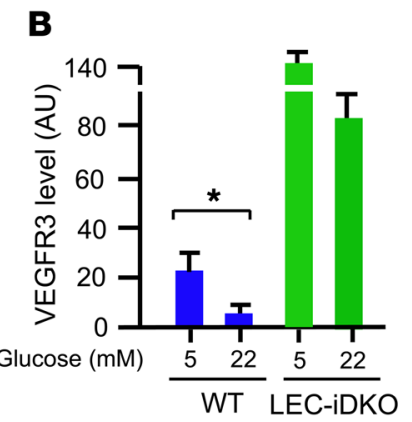

D
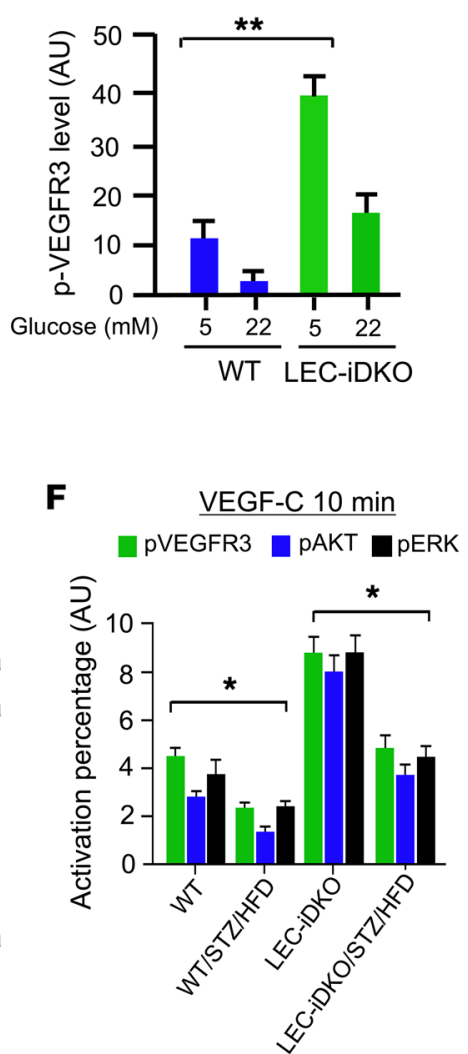

Figure 4. Epsin deficiency limits VEGFR3 internalization and increases surface abundance of VEGFR3 in LECs via VEGF-C signaling in diabetes. (A) Western blot analysis of surface biotinylated VEGFR3 in LECs of WT or LEC-iDKO. Cell-surface VEGFR3 was biotinylated then detected by pull-down assay with streptavidin beads and Western blotting with antiVEGFR 3 antibody. Integrin- $\alpha 9$ was used as membrane control. (B) Quantification of phosphorylation of VEGFR3 level in A. (C) Western blot analysis of p-VEGFR3, epsin 1 , and epsin 2 protein levels in LECs of WT or LEC-iDKO mice cultured in $5 \mathrm{mM}$ glucose or $22 \mathrm{mM}$ glucose for 5 days in the presence of $100 \mathrm{ng} / \mathrm{mL}$ VEGF-C. (D) Quantification of phosphorylation of VEGFR3 levels in C. (E) Western blot for VEGFR signaling in LECs from WT, LEC-iDKO, WT/STZ/HFD, and LEC-iDKO/STZ/HFD mice stimulated with VEGF-C at 0 and 10 minutes. (F) Quantification of activation percentages of phosphorylated VEGFR3, AKT, and ERK in $\mathbf{E}$. (G) Immunofluorescence staining of VEGFR3 (green) and DAPI (blue) in WT, LEC-iDKO, WT/STZ/HFD, and LEC-iDKO/ STZ/HFD LECs serum-starved overnight and stimulated with $100 \mathrm{ng} / \mathrm{mL}$ VEGF-C for 15 minutes. Scale bar: $20 \mu \mathrm{m}$. (H) Quantification of fluorescence intensity of cellsurface VEGFR3 and internalized VEGFR3 in G. Quantification was performed using $\mathrm{NIH}$ Imagel for p-VEGFR3, p-AKT, p-ERK levels, and the fluorescence intensity of VEGFR3 on the cell surface and VEGFR3 internalized in cytosolic regions. Data are mean \pm SEM, $n=5$. ${ }^{*} P<0.05$; ${ }^{*} P<0.01$, by 2-way ANOVA followed by Tukey's post hoc test.
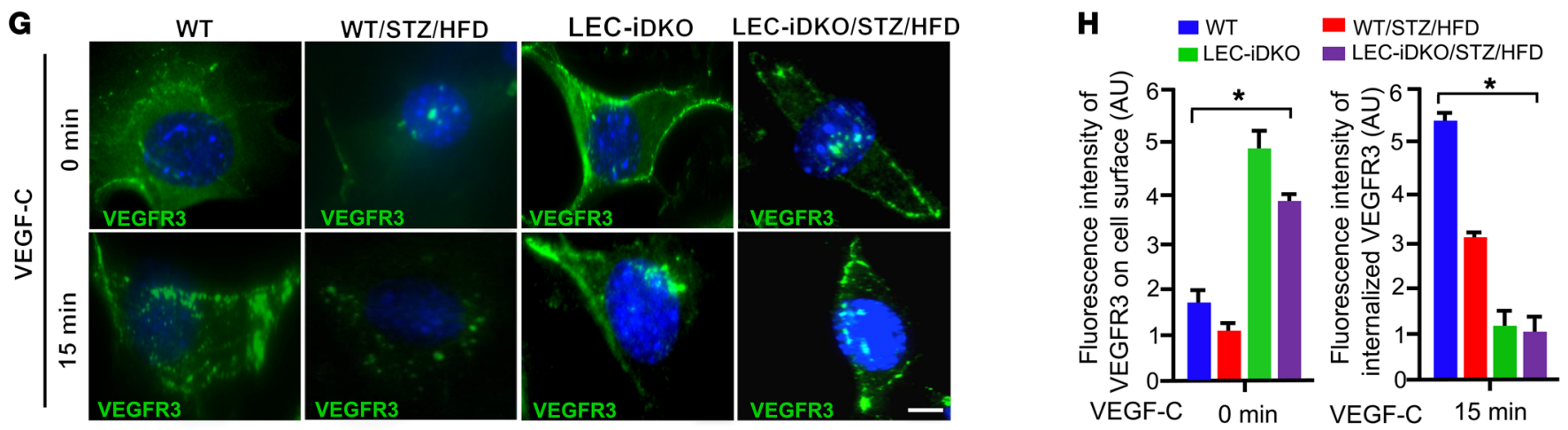

upper panel; Supplemental Figure 7F), suggesting that high levels of ROS activate $\mathrm{c}$-Src via phosphorylation in our STZ/HFD-induced type 2 diabetic mouse model, consistent with previous reports (50).

Next, we used a luciferase reporter assay to explore the transcriptional regulation of epsins in nondiabetic WT and diabetic
WT/STZ/HFD mice. A fragment of the epsin 1 promoter region containing the putative AP-1 binding sequence was inserted into the luciferase reporter vector pGL4.23[luc2/minP] in the sense orientation. To test if AP-1 is required for diabetes-induced epsin 1 expression, mutations were created in the AP-1 binding sites 
within the epsin 1 promoter fragment-driven luciferase reporter plasmids. These WT and mutant reporter plasmids were cotransfected with AP-1 expression plasmids or empty vectors into primary LECs freshly isolated from WT or WT/STZ/HFD mice. Two days after transfection, epsin 1 promoter activity was measured by the luciferase assay. To test whether diabetes-induced c-Src activation drives AP-1-dependent epsin 1 expression, we transfected c-Src siRNA to knock down the c-Src gene in primary mouse LECs (Figure 3F; Supplemental Figure 7F) prior to luciferase assay. Strikingly, relative luciferase activity of the WT construct in LECs from diabetic WT/STZ/HFD mice was much higher than that from control nondiabetic WT mice (Figure 3G). Furthermore, activity of the epsin 1 promoter containing the mutant AP- 1 binding site was suppressed more than $80 \%$ compared with the WT epsin 1 promoter activity (Figure 3G, green columns), indicating that hyperglycemia-induced epsin upregulation is AP-1 dependent. Consistently, luciferase activities in LECs of $d b / d b$ mice were remarkably increased compared with WT mice, and the luciferase activities of mutated AP-1 binding sites in the epsin 1 promoter were significantly decreased (Supplemental Figure 7D). Additionally, an AP-1 blocking peptide reduced the luciferase activities in LECs from both WT and WT/STZ/HFD mice, suggesting that the transcription factor AP-1 may bind to the epsin 1 promoter (Figure $3 \mathrm{H})$. Under c-Src knockdown conditions, the WT epsin 1 promoter activity in LECs from diabetic mice reverted back to the level of LECs from nondiabetic control mice (Figure 3G), demonstrating that AP-1-dependent upregulation of epsin requires c-Src.

To further confirm whether AP-1 can directly bind to the epsin 1 promoter through AP-1 binding sites, we performed a ChIP assay in LECs from both WT and WT/STZ/HFD mice with and without c-Src knockdown. The ChIP assay revealed a prominent increase in the association of AP- 1 with the epsin 1 gene promoter in the LECs from diabetic WT/STZ/HFD mice compared with the control WT mice (Figure 3I). In addition, the association of AP- 1 with the epsin 1 gene promoter in LECs from WT/STZ/ HFD mice was decreased under c-Src knockdown conditions (Figure 3I), consistent with our observations using the luciferase reporter assay (Figure 3, $\mathrm{G}$ and $\mathrm{H}$ ).

Collectively, our findings demonstrate that diabetic oxidative stress upregulates expression of epsins through c-Src-mediated and AP-1-driven transcriptional machinery.

Epsin deficiency heightens VEGF-C/VEGFR3 signaling by inhibiting VEGFR3 internalization and increasing surface abundance of VEGFR3 in LECs in diabetes. It remains unclear whether depletion of epsins in pathological conditions such as diabetes limits VEGFR3 lost under hyperglycemia and improves VEGF-C/VEGFR3 signaling in lymphatic endothelial cells, leading to increased lymphangiogenesis. To determine this, we treated LECs from WT and LEC-iDKO mice with different concentrations of glucose and stimulated them with VEGF-C. Cell-surface biotinylation assays revealed a marked decrease in the surface abundance of VEGFR3 in primary LECs from WT mice cultured in high-glucose medium (22 $\mathrm{mM})$ compared with low-glucose medium $(5 \mathrm{mM})$ for a minimum of 5 days (Figure 4, A and B). However, when LECs from LEC-iDKO mice were exposed to high glucose, depletion of epsins inhibited the decrease in surface abundance of VEGFR3 and phosphorylation of VEGFR3 compared with LECs from WT mice
(Figure 4, A-D). Additionally, in high-glucose medium, VEGFR3 downstream signaling, as measured by the phosphorylation of protein kinase B (p-AKT), was increased in LEC-iDKO cells compared with that in WT cells (Figure 4, C and D).

To corroborate these findings in acute hyperglycemic conditions, we measured VEGF-C/VEGFR3 signaling in chronic hyperglycemic conditions using freshly isolated LECs from WT, LEC-iDKO, WT/STZ/HFD, and LEC-iDKO/STZ/HFD mice. Consistently, STZ/HFD-induced hyperglycemia reduced total VEGFR3 protein abundance in WT/STZ/HFD mice but not in LEC-iDKO/STZ/HFD mice (Figure 4, E and F). After 10 minutes of stimulation with VEGF-C, LEC-iDKO cells exhibited increased levels of phosphorylated VEGFR3 and activation of downstream signaling components, including p-AKT and extracellular signalregulated kinase (p-ERK), compared with all other mice genotypes. These results are consistent with the aforementioned results under acute hyperglycemic conditions. In addition, VEGFC/VEGFR3 signaling in WT/STZ/HFD cells was decreased compared with other mice, whereas VEGF-C/VEGFR3 signaling in LEC-iDKO/STZ/HFD cells increased compared with that of WT/ STZ/HFD cells, but was similar to that of WT cells. This suggests that depletion of epsins may prevent degradation of VEGFR3, resulting in enhanced VEGF-C/VEGFR3 signaling under hyperglycemic conditions (Figure 4, $\mathrm{E}$ and $\mathrm{F}$ ).

We next examined whether the diminished degradation of VEGFR3 due to loss of epsins increases the cell-surface abundance of VEGFR3 in diabetic conditions. Notably, before treatment with VEGF-C, the most abundant levels of VEGFR3 (green fluorescence) on the cell surface were detected in LEC-iDKO cells compared with those on the cell surfaces of WT, WT/STZ/HFD, and LEC-iDKO/STZ/HFD cells (Figure 4, G and H). Furthermore, the abundance of VEGFR3 on LEC-iDKO/STZ/HFD cells was increased compared with that on WT/STZ/HFD cells. After 15 minutes of VEGF-C treatment, the green puncta of VEGFR3 were dramatically increased in the cytosolic region of WT and WT/STZ/HFD cells compared with those in LEC-iDKO and LECiDKO/STZ/HFD cells, respectively, suggesting that VEGF-Cinduced VEGFR3 internalization is epsin dependent. Importantly, cell-surface abundance of VEGFR3 in LEC-iDKO/STZ/HFD cells was still increased compared with WT/STZ/HFD cell-surface VEGFR3 levels.

Taken together, these data support a mechanism in which epsin deficiency heightens cell-surface levels of VEGFR3 and increases VEGF-C/VEGFR3 signaling in LECs from diabetic LECiDKO/STZ/HFD mice (Figure 4, G and H).

ROS-induced phosphorylation of VEGFR3 is c-Src dependent and ligand independent. Oxidative stress has been shown to contribute to endothelial dysfunction in diabetes (10), and has been considered a major hallmark of pathogenesis and development of type 2 diabetes mellitus. Little is known about how chronic hyperglycemia leads to reduction of cell-surface abundance of VEGFR3. Thus, we sought to explore the mechanisms by which elevated ROS leads to decreased abundance of VEGFR3 (Figure 4). Relatively high doses of ROS, such as $\mathrm{H}_{2} \mathrm{O}_{2}$ treatment, cause cell death by activating cell death signaling cascades $(10,51)$; however, proper dosage of oxidative stress can also activate certain cell signaling pathways that prevent cell death (52). VEGFR3 has been shown to serve as a cell sur- 
A

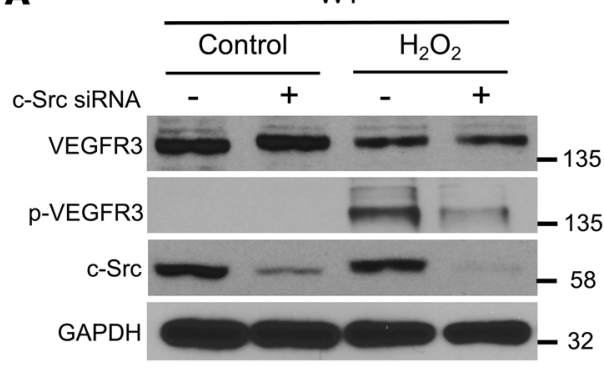

C

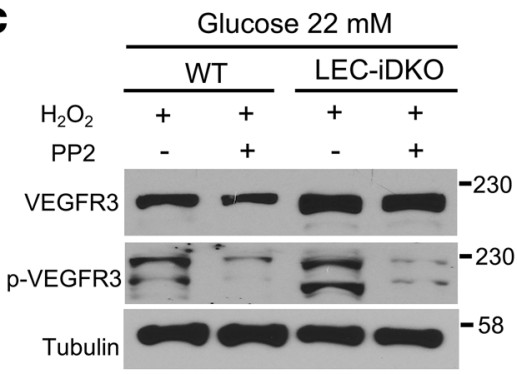

E

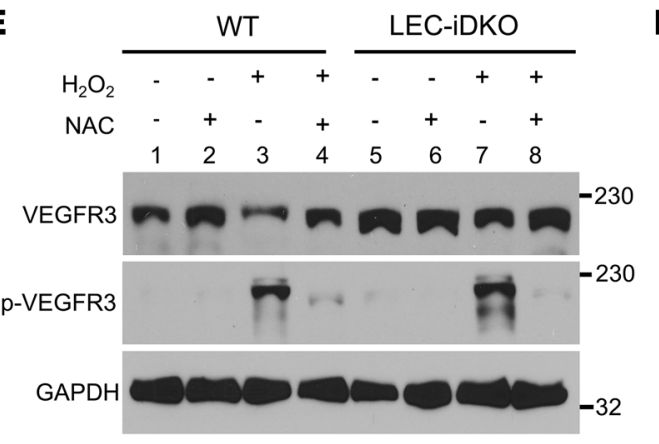

B

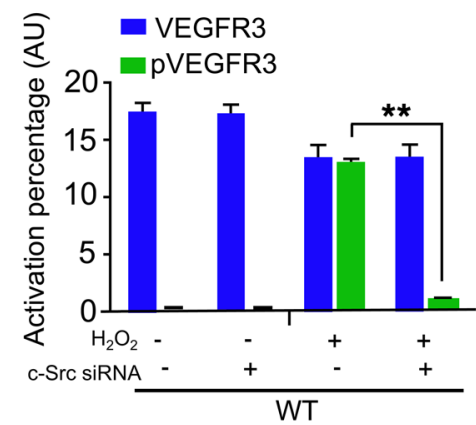

D

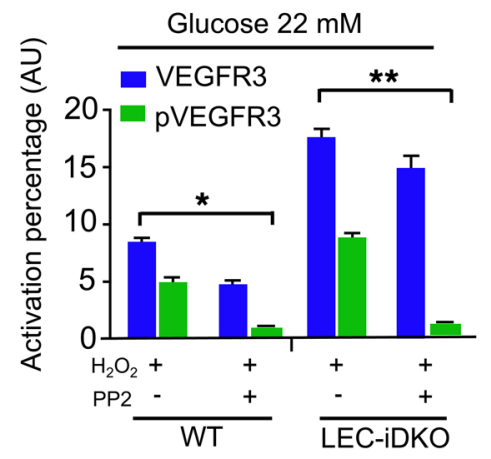

$\mathbf{F}$

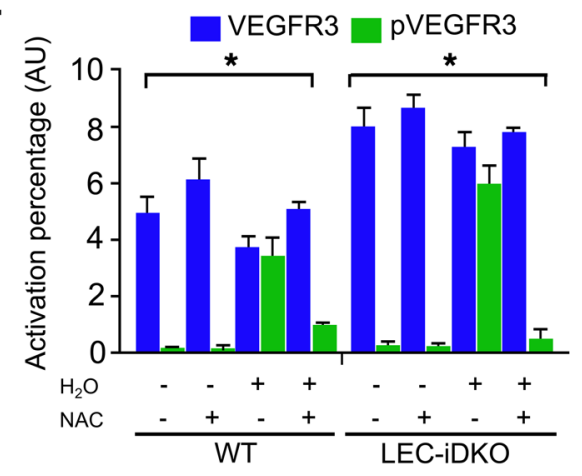

Figure 5. ROS-induced intracellular phosphorylation of VEGFR3 is c-Src dependent and ligand independent. (A) WT LECs were treated with control or c-Src siRNA followed by treatment with $\mathrm{H}_{2} \mathrm{O}_{2}$ and analysis via Western blot $(n=3)$. (B) Quantification of activation percentage of VEGFR3 and phosphorylation of VEGFR3 in $\mathbf{A}$. (C) WT and LEC-iDKO LECs were treated with $\mathrm{H}_{2} \mathrm{O}_{2}$ to induce phosphorylation of VEGFR3, PP2 to inhibit c-Src, and $22 \mathrm{mM}$ glucose followed by analysis using Western blot $(n=3)$. (D) Quantification of activation percentage of VEGFR3 and phosphorylation of VECFR3 in C. (E) Western blot analysis of phosphorylation of VEGFR3 abundance in LECS of WT and LEC-iDKO treated with $5 \mathrm{mM} \mathrm{N}$-acetyl-L-cysteine (NAC) for 30 minutes, and then treated with $500 \mu \mathrm{M}$ hydrogen peroxide $\left(\mathrm{H}_{2} \mathrm{O}_{2}\right)$ for 15 minutes $(n=7)$. (F) Quantification of activation percentage of VEGFR3 and phosphorylation of VEGFR3 in E. (G) Western blot analysis of $\mathrm{H}_{2} \mathrm{O}_{2}$-induced phosphorylation of VEGFR3 was protected from trypsin digestion. WT and LECiDKO LECs were briefly exposed to $0.25 \%$ trypsin for cell-surface proteolysis, and then treated with $500 \mu \mathrm{M} \mathrm{H}_{2} \mathrm{O}_{2}$ or 200 $\mathrm{ng} / \mathrm{mL}$ VEGF-C for 15 minutes prior to lysis. VE-cadherin is shown as membrane control. VE-cadherin and tubulin were run on the same gel but were noncontiguous $(n=7)$. (H) Quantification of activation percentage of phosphorylation of VEGFR3 in G. Quantification was done with NIH Image. Data are mean \pm SEM. ${ }^{*} P<0.05$; ${ }^{* *} P<0.01$, by 2 -way ANOVA (B, D, and F) or 3-way ANOVA (H) followed by Tukey's post hoc test.
G

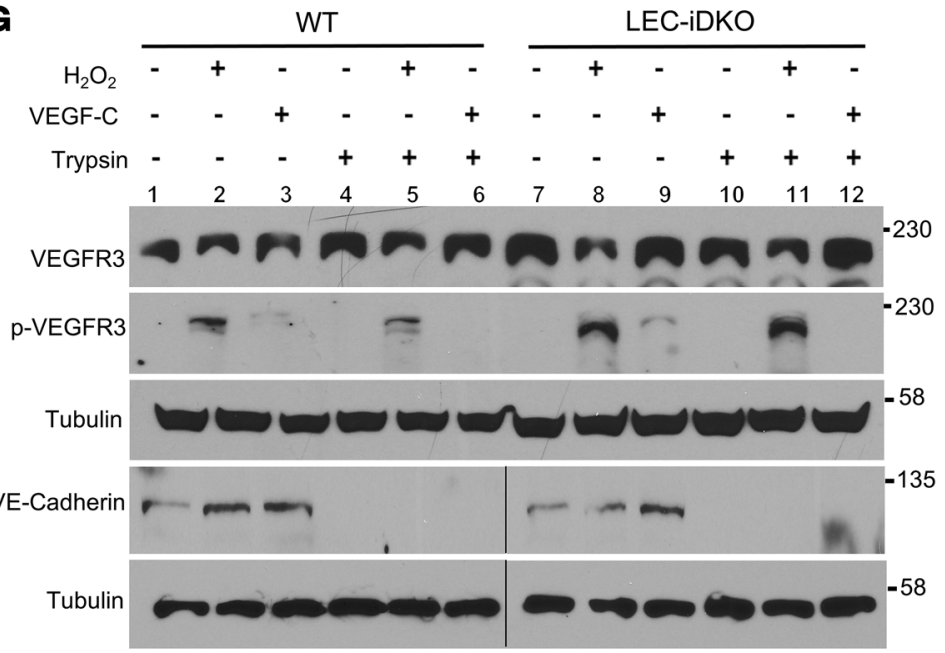

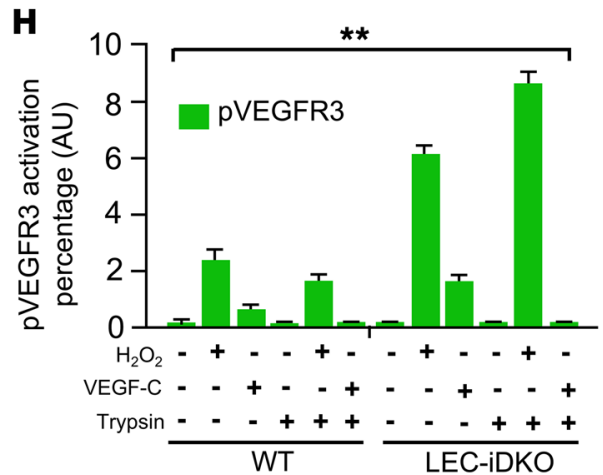

vival modulator under oxidative stress by functionally transmitting its downstream signaling (52). Based on our observation that epsins and VEGFR3 are inversely regulated in hyperglycemia, and that no significant difference is found in VEGFR3 mRNA levels between nondiabetic and diabetic conditions (Figure 3B), we posit that high
ROS-induced c-Src activation may cause VEGFR3 phosphorylation and result in its degradation independent of ligand binding. Strikingly, $\mathrm{H}_{2} \mathrm{O}_{2}$ induced phosphorylation of VEGFR 3 in freshly isolated mouse LECs (Figure 5, A and B). This was significantly reduced in c-Src knockdown cells, suggesting that ROS-induced VEGFR3 
phosphorylation is mediated by c-Src kinase (Figure 5, A and B). Additionally, the c-Src kinase family inhibitor PP2 hindered $\mathrm{H}_{2} \mathrm{O}_{2}$ induced VEGFR3 phosphorylation and degradation (Figure 5, C and D). Furthermore, depletion of epsins limited the decrease of VEGFR3 induced by $\mathrm{H}_{2} \mathrm{O}_{2}$ compared with that in WT LECs (Figure $5, \mathrm{C}$ and D). Interestingly, the phosphorylation levels of VEGFR3 induced by $\mathrm{H}_{2} \mathrm{O}_{2}$ in both WT and LEC-iDKO LECs were similar (Figure 5, E and F), indicating that epsins do not impact ROSinduced c-Src-mediated VEGFR3 phosphorylation. Furthermore, treatment with the $\mathrm{H}_{2} \mathrm{O}_{2}$ scavenger $\mathrm{N}$-acetyl-L-cysteine (NAC) was sufficient to reduce the phosphorylation of VEGFR3 induced by $\mathrm{H}_{2} \mathrm{O}_{2}$ in both WT and LEC-iDKO LECs to similar degrees (Figure 5, $\mathrm{E}$ and $\mathrm{F}$ ). Thus, ROS promotes phosphorylation of VEGFR3 regardless of the presence of epsins.

To further dissect the mechanism of ROS-induced intracellular phosphorylation of VEGFR3, and to definitively show that this type of phosphorylation is independent of ligand stimulation, we employed mild trypsin digestion to remove the extracellular ligand binding domain of VEGFR3 and tested $\mathrm{H}_{2} \mathrm{O}_{2}$-induced phosphorylation of VEGFR3 in the presence or absence of VEGF-C. After treatment with $0.25 \%$ trypsin to cleave the extracellular domains of cell-surface proteins, freshly isolated LECs from WT and LECiDKO mice were treated with or without $\mathrm{H}_{2} \mathrm{O}_{2}$ and stimulated with or without VEGF-C. Relative to VEGF-C-stimulated activation of VEGFR3 (lanes 3 and 9), $\mathrm{H}_{2} \mathrm{O}_{2}$ induced more profound VEGFR3 phosphorylation (lanes 2 and 8) (Figure 5, G and H). Due to the removal of extracellular domains by trypsin digestion, phosphorylation of VEGFR3 induced by VEGF-C was completely abolished in both WT (lane 6) and LEC-iDKO (lane 12) (Figure 5, G and H). Trypsin cleavage also abolished detectable VE-cadherin when an antibody against the VE-cadherin extracellular domain was used as a control (Figure 5G). However, VEGFR3 phosphorylation in response to $\mathrm{H}_{2} \mathrm{O}_{2}$ was resistant to extracellular trypsin digestion, and phosphorylation of VEGFR3 was seen in both WT (lane 5) and LEC-iDKO (lane 11), suggesting ROS-induced phosphorylation of the intracellular VEGFR3 is ligand independent (Figure 5, G and H).

Taken together, these results indicate that oxidative stress can induce ligand-independent phosphorylation of VEGFR3 via activation of c-Src kinase.

ROS induces VEGFR 3 association with epsins within the Golgi under diabetic conditions. Previous studies have shown that high-glucose exposure induces oxidative stress, resulting in the intrinsic intracellular phosphorylation of VEGFR2 in the Golgi and subsequent VEGFR2 degradation, leading to endothelial dysfunction (28). We hypothesized that hyperglycemia-induced oxidative stress causes the intrinsic phosphorylation of VEGFR3 in the Golgi compartment and induces recruitment of epsins to the Golgi compartment where the epsins bind VEGFR3 and mediate its subsequent trafficking to the lysosome for degradation in the lymphatic endothelium.

To test this hypothesis, we examined whether VEGFR3 is phosphorylated by $\mathrm{H}_{2} \mathrm{O}_{2}$ in the Golgi compartment by performing immunofluorescence staining of total and phosphorylated VEGFR3 or epsin 1 with the Golgi marker GM130 after treatment with or without $\mathrm{H}_{2} \mathrm{O}_{2}$. Immunofluorescence costaining revealed that total and intrinsically phosphorylated levels of VEGFR3 largely colocalized with GM130-positive Golgi when WT LECs were exposed to $\mathrm{H}_{2} \mathrm{O}_{2}$, whereas the colocalization of total VEG-
FR3 with GM130 was reduced with minimal phosphorylation of VEGFR3 in control-treated cells (Figure 6, A and B). Interestingly, the colocalization between epsin 1 and GM130 was enhanced by $\mathrm{H}_{2} \mathrm{O}_{2}$ stimulation, suggesting that epsins are localized to the Golgi compartment in response to $\mathrm{H}_{2} \mathrm{O}_{2}$ treatment (Figure 6, A and $\mathrm{B}$ ). Next, we performed the same experiments with LECs isolated from WT/STZ/HFD and $d b / d b$ diabetic mice under hyperglycemic conditions. Immunofluorescence data showed that total and intrinsically phosphorylated VEGFR3 largely colocalized with GM130-positive Golgi in LECs from both WT/STZ/HFD diabetic mice (Figure 6, A and B) and $d b / d b$ diabetic mice (Supplemental Figure 8). Total and phosphorylated VEGFR3 colocalization was increased compared with LECs from the WT control mice. The colocalization between epsin 1 and GM130 was also enhanced in WT/STZ/HFD and $d b / d b$ mice (Figure 6, A and B and Supplemental Figure 8). These results are consistent with our observations in LEC cells from WT mice treated with $\mathrm{H}_{2} \mathrm{O}_{2}$ (Figure 6), suggesting that ROS induces VEGFR3 and epsin association with epsins within the Golgi under diabetic conditions. Interestingly, immunofluorescence staining revealed that epsin 2 also colocalizes with VEGFR3 in the Golgi under hyperglycemic conditions in $d b / d b$ mice, indicating that both epsins 1 and 2 are involved in the epsin-dependent mechanism of impaired lymphangiogenesis in the context of diabetes (Supplemental Figure 8).

To determine whether epsins and VEGFR3 form a complex in the Golgi compartment in response to hyperglycemia, we coimmunoprecipitated VEGFR3 with epsin 1. WT LECs were treated with or without $\mathrm{H}_{2} \mathrm{O}_{2}$ for 30 minutes, lysed, and fractionated to isolate Golgi-enriched fractions, followed by immunoprecipitation with anti-epsin 1 antibodies. Strikingly, the phosphorylation of VEGFR3 was prominent in Golgi-enriched fractions from LECs treated with $\mathrm{H}_{2} \mathrm{O}_{2}$. This enhanced phosphorylation of VEGFR3 in Golgienriched fractions was positively correlated with the increased coimmunoprecipitation of VEGFR3 with epsin 1 (Figure 6C).

Confocal immunofluorescence images of LECs from both WT/STZ/HFD and LEC-iDKO/STZ/HFD mice demonstrated that VEGFR3 was associated with the lysosome marker LAMP1 to a greater extent in those LECs than in LECs of WT and LEC-iDKO mice. Moreover, the colocalization of VEGFR3 and LAMP1 was reduced in LEC-iDKO/STZ/HFD LECs compared with WT/STZ/ HFD LECs (Supplemental Figure 9).

Taken together, these data suggest that under diabetic conditions, $\mathrm{H}_{2} \mathrm{O}_{2}$ or high $\mathrm{ROS}$ enhances the recruitment of epsins to the Golgi compartment as well as the binding of epsins to phosphorylated VEGFR3 to trigger its lysosomal degradation. However, deficiency of epsins can prevent VEGFR3 degradation in the lysosome under diabetic conditions.

Epsin deficiency reinstates insufficient lymphangiogenesis in vivo and improves the resolution of tail edema in diabetic mice. The mouse tail edema model is a well-established model to study lymphangiogenesis in skin regeneration $(11,53)$. Given the critical role of epsins in VEGFR3-mediated lymphangiogenesis, we hypothesized that deficiency of epsins could promote lymphangiogenesis and rescue impaired lymphatic transport by downregulating VEGFR3 degradation in diabetes. To test our hypothesis, we established a tail edema model in our WT, LEC-iDKO, WT/STZ/ HFD, and LEC-iDKO/STZ/HFD mice by incising the circumfer- 
A
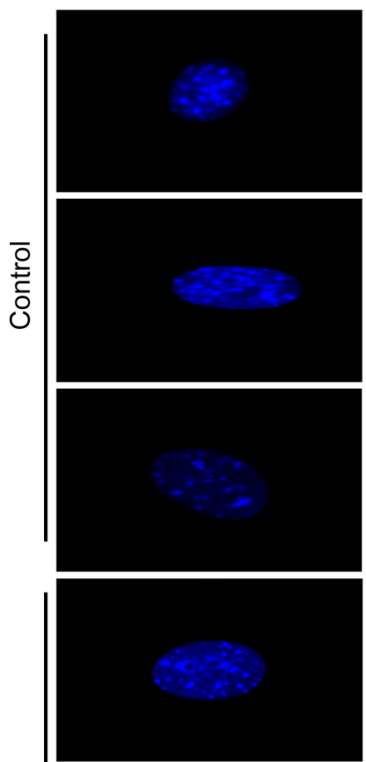

$\mathrm{O}^{N}$
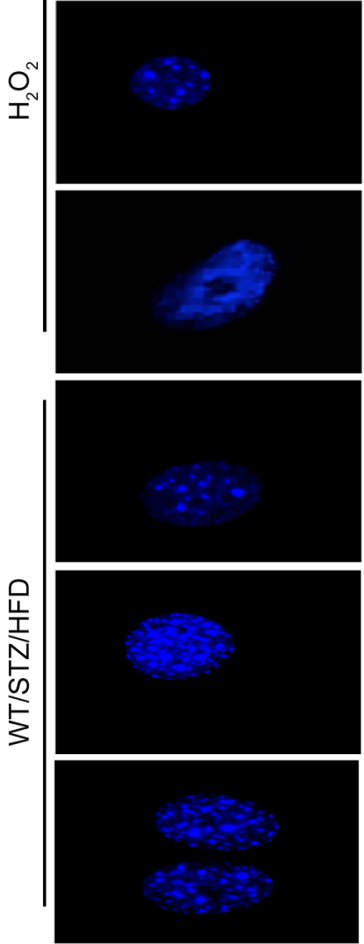

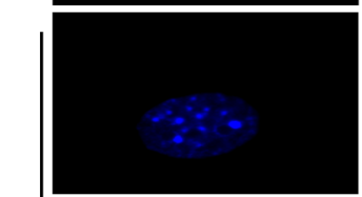

Alexa Fluor-488
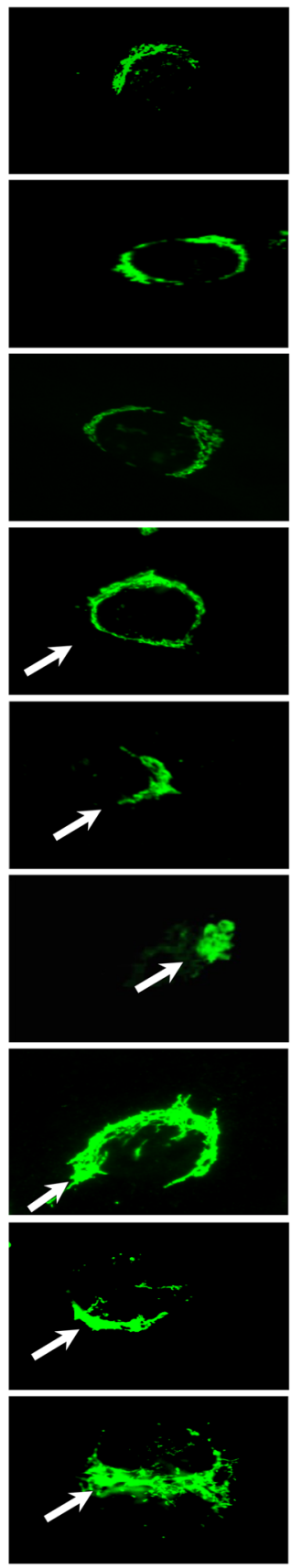

Alexa Fluor-594
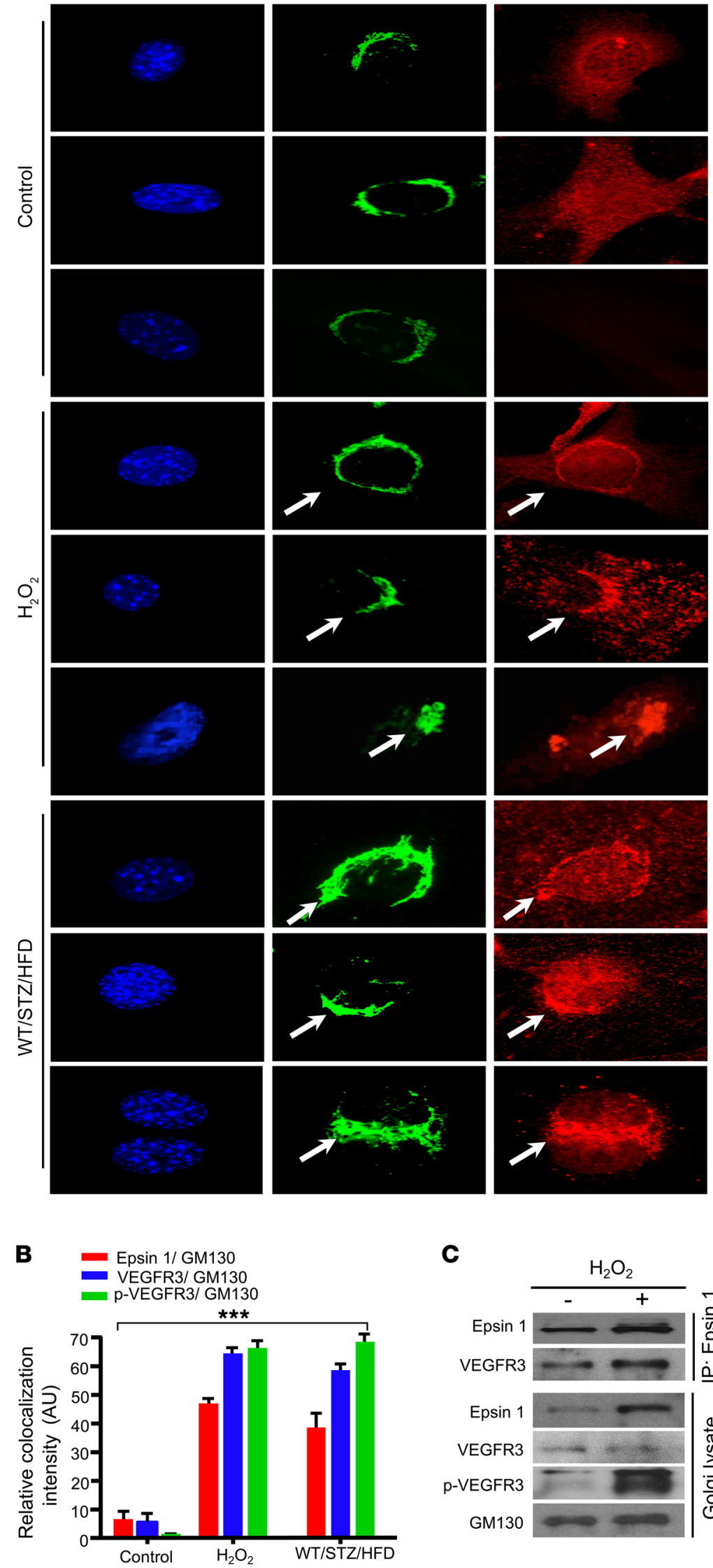

C

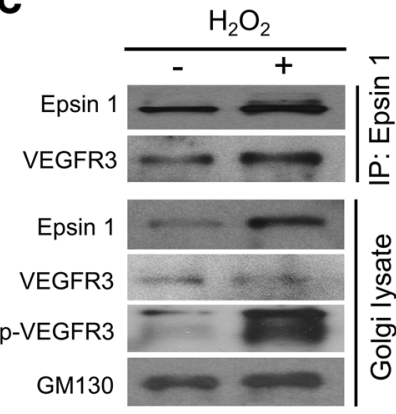

Merge
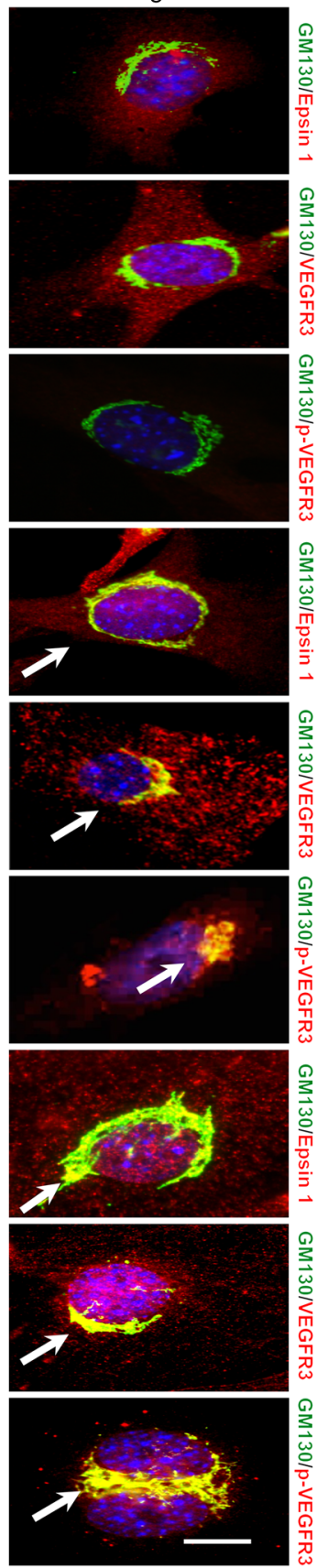

Figure 6. ROS-induced phosphorylation of VEGFR3 associated with epsins in the Golgi compartment under diabetic conditions. (A and B) LECs isolated from WT control mice treated with $500 \mu \mathrm{M}$ hydrogen peroxide $\left(\mathrm{H}_{2} \mathrm{O}_{2}\right)$ for 15 minutes, or LECs isolated from WT/STZ/HFD mice, were stained with DAPI (blue) and GM130 (green); epsin 1 (red), GM130 (green), and VEGFR3 (red); or GM130 and phosphorylated VEGFR3 (red). Scale bar: $20 \mu \mathrm{m}$. (C) WT LECs treated with $\mathrm{H}_{2} \mathrm{O}_{2}$ were fractionated to isolate the Golgi compartment, which was further processed for immunoprecipitation of VEGFR3 using anti-epsin 1 antibodies and analyzed using Western blot. Arrows indicate spots of colocalization. Quantifications performed using NIH ImageJ. Data are mean \pm SEM, $n=5$. ${ }^{* *} P<0.001$, by 1 -way ANOVA followed by Tukey's post hoc test. 
ence of the tail base dermis, which effectively disrupted the initial and collecting lymphatic vessels. Predictably, WT/STZ/HFD mice experienced the heaviest tail edema and retarded healing after wounding, whereas LEC-iDKO mice experienced the least tail edema and most rapid healing after wounding (Figure 7A). However, both WT and LEC-iDKO/STZ/HFD mice exhibited similar levels of tail edema and healing time (Figure 7, A and B, and Supplemental Figure 10A), suggesting that hyperglycemia greatly impaired reparative lymphangiogenesis and deficiency of epsins ameliorated this defective lymphangiogenesis. It is known that lymphedema tissues show elevated levels of VEGF-C (15). To investigate whether lymphangiogenesis observed in the tail edema model in LEC-iDKO and LEC-iDKO/STZ/HFD mice is VEGF-C dependent, we measured VEGF-C levels in both serum and tail tissues. In the STZ/HFD diabetic models, we observed no significant change in VEGF-C levels in both the serum and tissues from WT, LEC-iDKO, WT/STZ/HFD, and LEC-iDKO/STZ/ HFD mice at postoperative day 14. This indicates that the STZ/ HFD diabetic models are different from the K14-VEGF-C transgenic mouse model, suggesting that lymphangiogenesis observed in the tail lymphedema of LEC-iDKO and LEC-iDKO/STZ/HFD is VEGF-C independent (Supplemental Figure 11).

We next examined lymphatic vessels in the hypodermis by immunofluorescence staining for LYVE-1 and VEGFR3, which revealed significantly increased lymphatic vessel formation in the LEC-iDKO mice compared with WT mice (Figure 7, C and D). Lymphatic vessels in LEC-iDKO/STZ/HFD mice were also markedly increased compared with those in WT/STZ/HFD mice, supporting the conclusion that deficiency of epsins enhances lymphangiogenesis, leading to the improved resolution of tail edema. Lipid accumulation is a chronic indicator of tail edema (54). Oil Red O staining of tail sections from WT, WT/STZ/HFD, LECiDKO, and LEC-iDKO/STZ/HFD mice showed that WT and WT/ STZ/HFD mice exhibited more sustained lipid accumulation in the hypodermis of the tail distal to the incision and had increased thickness compared with similar areas in LEC-iDKO and LECiDKO/STZ/HFD mice (Supplemental Figure 10, B and C). This suggests that loss of epsins in LECs promotes the resolution of tail edema. In addition, immunohistochemistry staining for LYVE-1 and VEGFR3 in skin lacking exogenous VEGF-C demonstrated ring complexes in WT, LEC-iDKO, WT/STZ/HFD, and LECiDKO/STZ/HFD mice. However, the VEGFR3 level in the overall lymphatic network of WT/STZ/HFD was significantly decreased compared with that of LEC-iDKO/STZ/HFD, suggesting that epsin deficiency promotes lymphangiogenesis by increasing the abundance of VEGFR3 in LECs under diabetic conditions (Supplemental Figure 12). Furthermore, LYVE-1 $1^{+}$cells examined by flow cytometry also indicated that lymphangiogenesis in LEC-iDKO/ STZ/HFD mouse skin was significantly higher than that in WT/ STZ/HFD mice (Supplemental Figure 13).

To elucidate whether deficiency of epsins ensures sufficient lymphatic drainage to allow efficient tail edema resolution in the aforementioned mice, we injected FITC-dextran into the tails of WT, WT/STZ/HFD, LEC-iDKO, and LEC-iDKO/STZ/HFD mice at postoperative day 30 and monitored lymph drainage across the incision. Although still edematous, the initial lymphatics in these mice were functional and absorbed the FITC-dextran from the interstitial space as visualized by the concentration of FITCdextran collected on the distal side of the incision (Figure 7, E and F). However, hyperglycemia greatly inhibited the FITC-dextran drainage across the tail incision in diabetic WT/STZ/HFD mice (Figure 7, E and F). Furthermore, deficiency of epsins in LECiDKO/STZ/HFD mice ameliorated the hyperglycemic effect and led to rapid FITC-dextran drainage comparable to euglycemic WT mice (Figure 7, E and F).

To further dissect the potential contribution of VEGFR2 in LECs during the epsin-dependent and ligand-independent mechanism of impaired lymphangiogenesis in the context of diabetes, we crossed the LEC-iDKO mice with VEGFR3-eGFP knockin mice $\left(F l t 4^{e G F P /+}\right)$, effectively creating mice heterozygous for VEGFR3 on an inducible LEC-specific epsins 1 and 2 deletion background (LEC-iDKO-Flt4 $\left.{ }^{\text {eGFP/+}}\right)(34,55)$. Furthermore, we repeated the tail edema study in these diabetic WT/STZ/HFD, LEC-iDKOFlt $^{e G F P /+} /$ STZ/HFD, and LEC-iDKO/STZ/HFD mice. As predicted, WT/STZ/HFD mice experienced the heaviest tail edema and delayed wound healing compared with both LEC-iDKO-Flt $4^{e G F P /+}$ / STZ/HFD and LEC-iDKO/STZ/HFD mice. However, LEC-iDKOFlt $^{\text {eGFP/+ }} /$ STZ/HFD mice exhibited significantly decreased wound healing compared with LEC-iDKO/STZ/HFD mice (Supplemental Figure 14, A and B). Thus, genetic reduction of VEGFR3 levels results in disruption of lymphangiogenesis in diabetic conditions without genetic modification of VEGFR2 in these mice. Western blot analysis showed that VEGFR3 levels in LECs from LEC-iDKOFlt $^{\text {eGFP/+}} /$ STZ/HFD mice were reduced compared with levels in LEC-iDKO/STZ/HFD mice, but increased compared with levels in WT/STZ/HFD mice. Importantly, VEGFR2 levels were nearly equal across these 3 groups of diabetic mice, but all were quantitatively less than relative VEGFR3 levels (Supplemental Figure 14, $\mathrm{C}$ and D). This is consistent with previous studies in which lower levels of VEGFR2 were detected in LECs than in blood endothelial cells (56), whereas VEGFR3 protein was predominantly expressed by LECs, indicating that any potential contribution of VEGFR2 in LECs is very limited in our diabetic models.

In addition, we also analyzed eNOS and NO production in LECs isolated from WT, LEC-iDKO, WT/STZ/HFD, and LECiDKO/STZ/HFD by Western blot and fluorescent indicator DAF2. The eNOS levels corresponded with phosphorylated VEGFR3 levels in LECs from WT, LEC-iDKO, WT/STZ/HFD, and LECiDKO/STZ/HFD mice (Supplemental Figure 15, A-C and Figure $4 \mathrm{E})$. NO production in LECs from WT/STZ/HFD mice as determined by DAF-2 were significantly reduced compared with LECs from LEC-iDKO and LEC-iDKO/STZ/HFD mice (Supplemental Figure 15D). We next investigated whether depletion of epsins can rescue lymphatic leakage in STZ/HFD diabetic models by measuring lymph flow in the ears of WT, LEC-iDKO, WT/STZ/ HFD, and LEC-iDKO/STZ/HFD mice. Evans blue visual lymphangiography and subsequent quantification were performed to follow lymph drainage patterns. WT/STZ/HFD mice exhibited significantly enhanced leakiness of lymphatic vessels and retrograde lymph flow compared with other groups (Supplemental Figures 15, 16). Taken together, our data demonstrate that depletion of epsins in LECs can significantly stabilize the eNOS-NO axis and prevent lymphatic leakage through the regulation of VEGFR3 degradation in diabetes. 
A

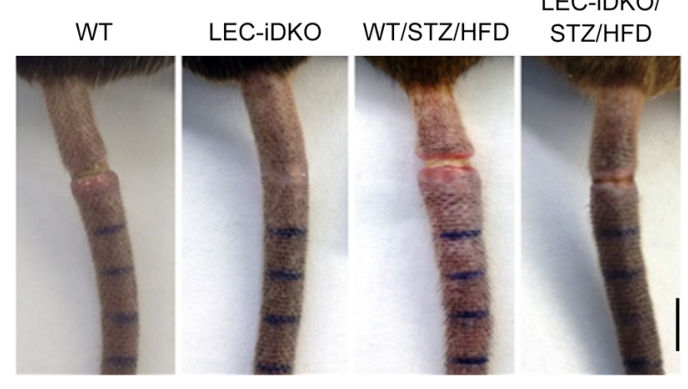

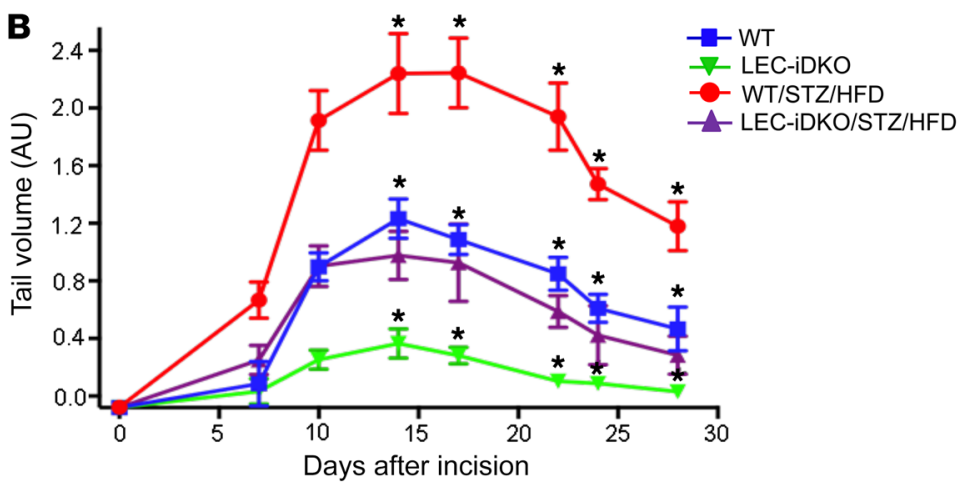

C
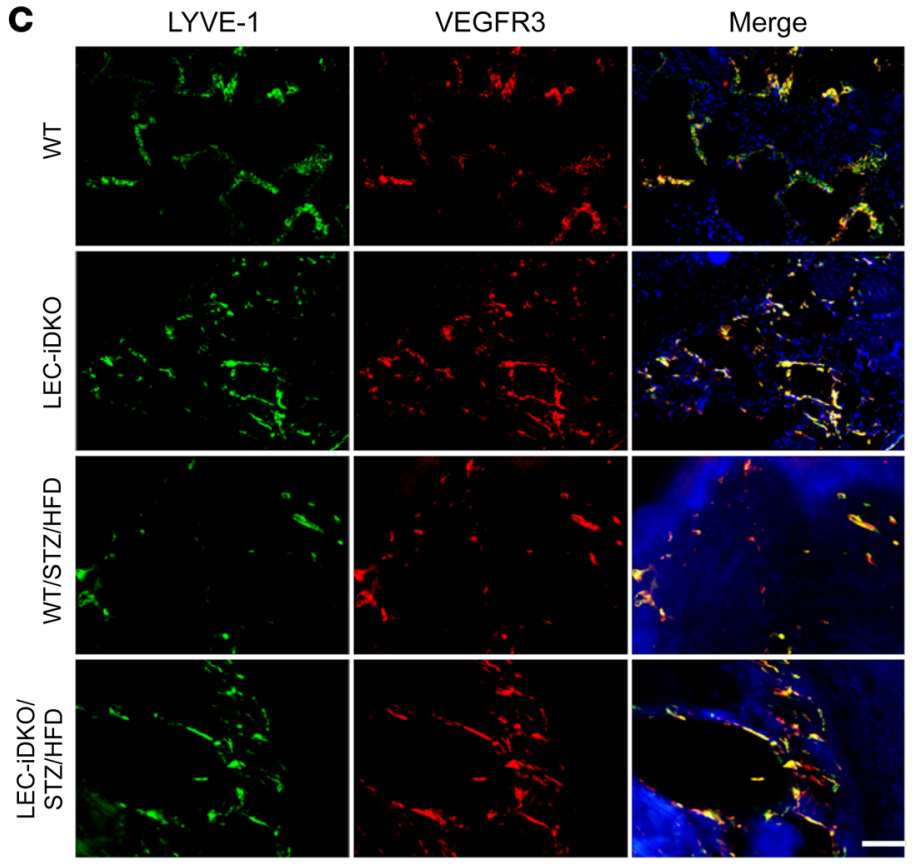

E
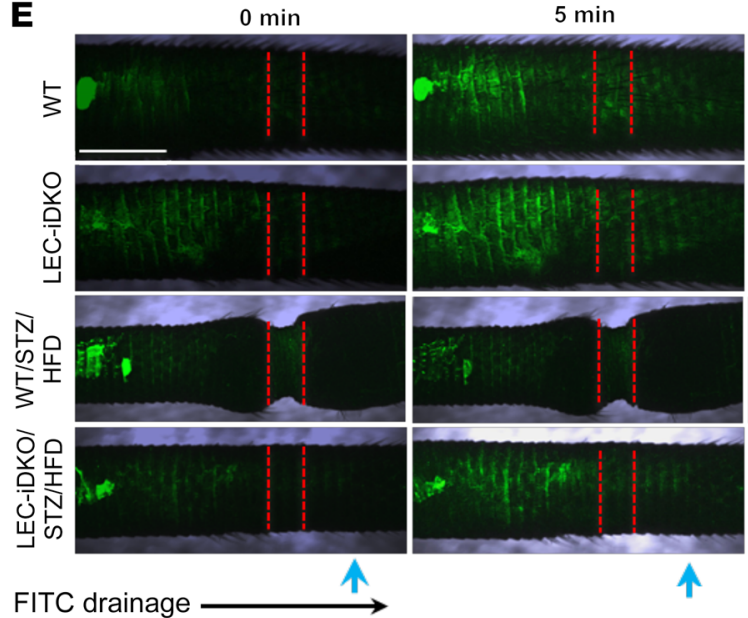

$\uparrow$
D
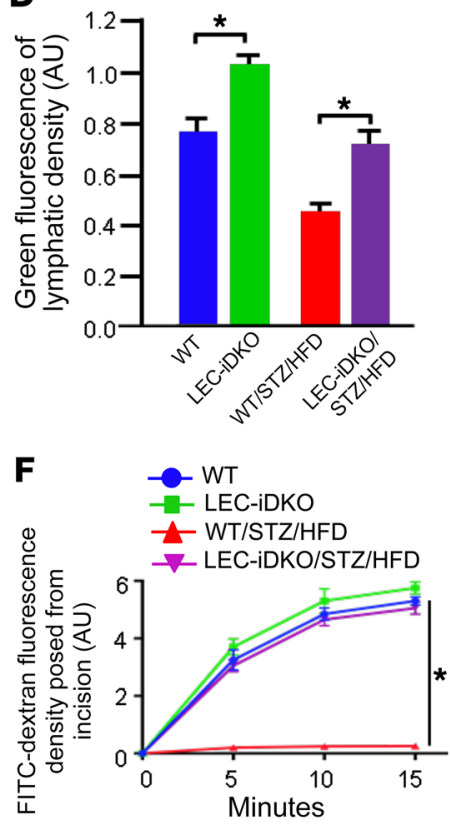

$15 \min$

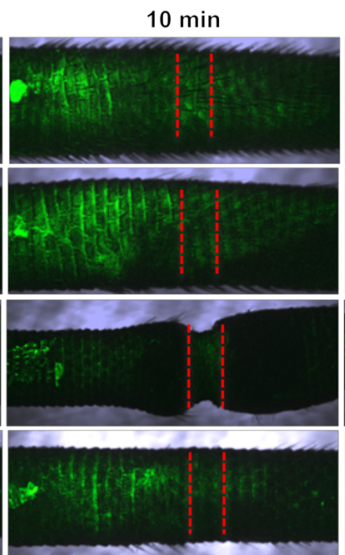

个

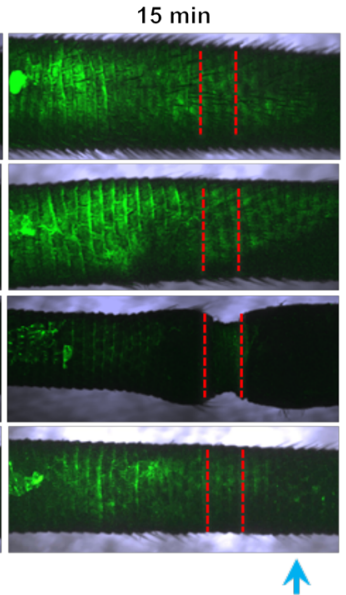

Figure 7. Epsin deficiency improves the resolution of tail edema by preventing the degradation of VECFR3 in diabetic mice. (A) Representative images of postoperative tail edema of WT $(n=8)$, LEC-iDKO $(n=8)$, WT/STZ/HFD $(n=8)$, and DKO/STZ/HFD $(n=8)$ mice at postoperative day 16. (B) Quantification of tail volume distal to the incision of postoperative tail edema of WT $(n=8)$, LEC-iDKO $(n=8)$, WT/STZ/HFD $(n=8)$, and DKO/STZ/HFD ( $n=8)$ mice at postoperative days $1,7,10,14,18,21,24$, and 28. (C and D) Representative immunofluorescence staining of tail sections of WT, LEC-iDKO, WT/STZ/HFD, and LEC-iDKO/STZ/HFD mice at postoperative day 29 with antibodies against LYVE-1 (green) and VEGFR3 (red). The quantification of immunofluorescence staining of tail sections of C is shown in $\mathbf{D}(n=5)$. ( $(\mathbf{a}$ and $\mathbf{F})$ Representative fluorescence microlymphangiography of FITC-dextran injection into tails of WT $(n=5)$, LEC-iDKO $(n=5)$, WT/STZ/ HFD $(n=5)$, and LEC-iDKO/STZ/HFD $(n=5)$ mice at postoperative day 30 , and analyzed by 3 independent experiments. Two red dashed lines indicate the incision of tails. Black arrow under the image marks the direction of lymphatic transport. Four cyan arrows indicate the FITC-dextran labeling fluorescence intensity after crossing the incision to other side of the tail at 0, 5, 10, and 15 minutes, respectively. Quantification of FITC-dextran labeling intensity posed after crossing the incision in F. Data are mean \pm SEM. ${ }^{*} P<0.05$, by 2-way ANOVA followed by Tukey's post hoc test. Scale bars: $8 \mathrm{~mm}(\mathbf{A}$ and $\mathbf{E}) ; 50 \mu \mathrm{m}(\mathbf{C})$. 


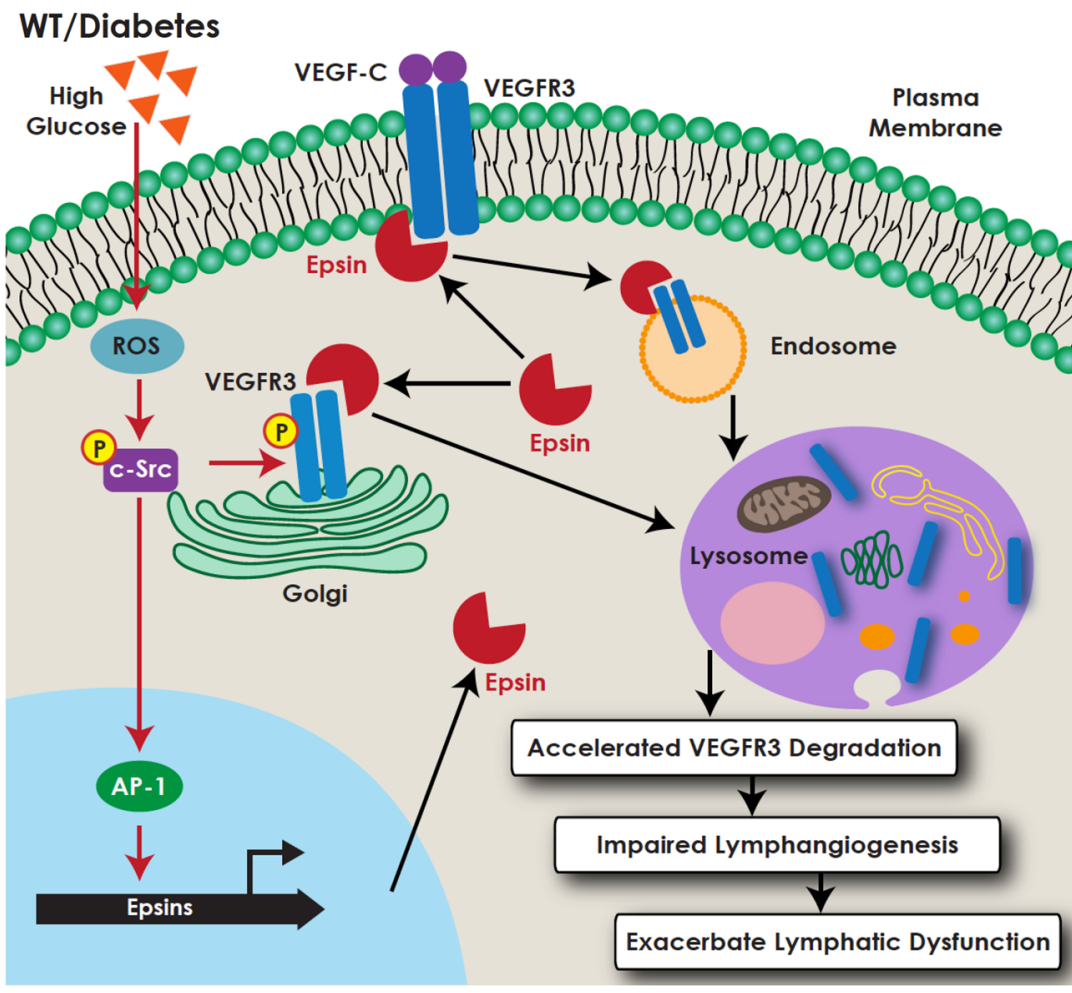

\section{LEC-iDKO/Diabetes}

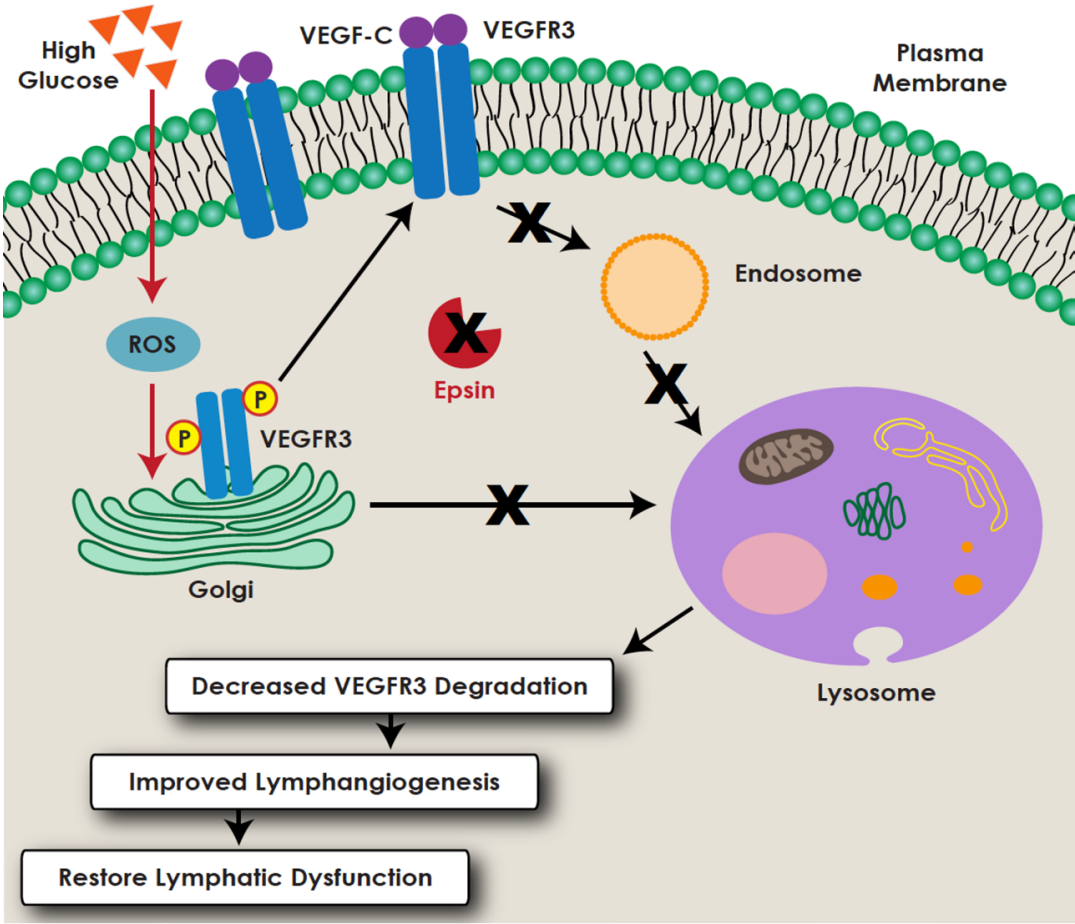

Figure 8. The model of epsin deficiency promotes lymphangiogenesis through regulation of VEGFR3 degradation in diabetes. In WT diabetic condition (upper panel), ROS generated from hyperglycemia causes c-Src-dependent VEGFR3 phosphorylation within the Golgi compartment. Heightened expression of epsins induced by ROS facilitates epsin binding to VEGFR3 in the Golgi, promotes newly synthesized VEGFR3 degradation, and progressively decreases availability of VEGFR3 at the cell surface. Accelerated VEGFR3 degradation can impair lymphangiogenesis, which exacerbates lymphatic function in WT diabetic mice. In the LEC-iDKO diabetic condition (lower panel), depletion of epsins results in attenuated VECFR3 degradation. The limited degradation of VEGFR3 maintains VEGFR3 surface abundance and sustains the robustness of VEGFR3 signaling to improve lymphangiogenesis, which can restore lymphatic function in LEC-iDKO diabetic mice.
In summary, our findings demonstrate that deficiency of epsins can promote lymphatic vessel growth in diabetes both in vivo and in vitro. Loss of epsins is beneficial in protecting against tail edema in diabetes by sustaining an adequate threshold of cell-surface VEGFR3 necessary to establish sufficient lymphangiogenesis and normalization of lymphatic regeneration. Mechanistically, ROS induces ligand-independent and c-Srcdependent VEGFR3 phosphorylation in the Golgi compartment. Meanwhile, ROS can also induce c-Src-dependent epsin upregulation through the transcription factor AP-1. Heightened expression of epsins facilitates the interaction between epsins and VEGFR3 within the Golgi, and promotes newly synthesized VEGFR3 degradation, resulting in progressively decreased availability of VEGFR3 at the cell surface (Figure 8).

\section{Discussion}

In this study, we report the novel discovery that hyperglycemia-induced oxidative stress intrinsically causes VEGFR3 for degradation independent of VEGF-C stimulation, and that loss of epsins may rescue diabetes-impaired lymphangiogenesis by prohibiting degradation of VEGFR3 in the Golgi compartment. Thus, deficiency of epsins allows maintenance of a sufficient level of cell-surface VEGFR3 that is readily responsive to VEGF-C stimulation, promoting normalized lymphangiogenesis under diabetic conditions.

Lymphatic systems are crucial in the pathogenesis of various diseases, including diabetes, obesity, and cancer (1, 39, 57-60). Excess lymphangiogenesis causes cancer metastasis and inflammation, and is associated with lymphatic hyperplasia in some forms of lymphedema (15, 61). Conversely, diminished or defective lymphangiogenesis also underlies or exacerbates disorders, including diabetes and lymphedema. Aberrant lymphatic vessel growth is observed in type 2 diabetes both clinically and in animal models, suggesting that defective lymphangiogenesis may contribute to diabetic complications such as persistent inflammation and edema in diabetic patients. However, the molecular mechanisms governing lymphangiogenesis in diabetes are poorly understood, despite extensive studies of LECs from human clinical samples, diabetic animal models, and a mathematical model $(7,38-40$, 62-64). Therefore, genetically manipulated animal models that directly influence lymphangiogenesis and display core characteristics of diabetic patients, especially type 2 diabetic patients who are more prevalent than type 1 diabetic patients, are imperative to address current challenges. We posited that these unique diabetic animal models, 
which selectively lack important regulators for lymphangiogenesis, would allow us to dissect the precise mechanisms underlying this complex disease and elucidate novel targeting strategies. Our present study employs a combination of STZ and HFD treatment in LEC-iDKO mice to achieve sustained hyperglycemic conditions typically found in type 2 diabetic patients. To substantiate our findings, future studies utilizing genetic diabetic mouse models, such as $d b / d b$ or $o b / o b$ mice that are bred to LEC-iDKO background, will be necessary. Likewise, whether epsins mediate the degradation of VEGFR3 via similar mechanisms in type 1 diabetes will be tested using analogous genetic mouse models, such as Akita mice, which are bred to LEC-iDKO background.

For the present study, we created a unique diabetic mouse model that utilized the inducible conditional LEC-iDKO mice driven by Prox1-Cre-ER ${ }^{T 2}$ (65), which differs from the constitutive LEC-DKO mice driven by Lyve-1-Cre (34). This allowed us to circumvent developmental defects in lymphatic systems owing to constitutive deletion of lymphatic endothelial epsins in the embryonic stage. This inducible LEC-iDKO mouse strain enables us to deplete epsins in adulthood, more specifically in the context of a well-developed and functional lymphatic system. These mice are grossly normal and we observed no abnormal lymphatic phenotypes associated with the deletion of epsins within the time frame of our studies. This suggests that conditionally targeting epsins under pathological conditions should result in minimal disruptions to the lymphatic homeostasis required for normal physiology. Furthermore, deficiency of epsins 1 and 2 does not impair housekeeping clathrin-mediated endocytosis, suggesting that epsins interact with their binding partners with a high degree of selectivity (32). Consistently, we previously reported that in endothelial cells, epsins 1 and 2 preferentially bind ubiquitinated VEGFR2 to mediate its lysosomal degradation without affecting other angiogenic receptors and their downstream signaling, including PDGFR, FGFR, EGFR, and TGF- $\beta$ R $(30,32,33)$. Therefore, we anticipate that the epsin binding partners in LECs are equally restricted and in fact, we reported that epsins interact with VEGFR3 in a similar fashion as VEGFR2 in LECs (34). Given a well-established role for epsins as endocytic adaptor proteins that are critical for ligand-triggered cell-surface VEGFR2 and VEGFR3 internalization and degradation $(34,66)$, our findings on epsins binding to VEGFR3 in the Golgi and mediating its degradation represent an unexpected new discovery and a novel function of epsins. A recent study showed that high glucose induced ligand- and intrinsic kinase-independent VEGFR2 phosphorylation in the Golgi, resulting in reduced VEGFR2 trafficking to the cell surface (28). Given the mechanistic similarity of epsin binding to VEGFR2 and VEGFR3, we posit that epsins act in a similar fashion to bind Golgiresiding VEGFR2 to mediate its degradation under hyperglycemic conditions. A documented role for VEGFR2 in promoting reparative neovascularization during wound healing has been proposed (35); however, whether VEGFR2 in LECs also contributes to the resolution of tail edema is unclear. Attempts to directly address this question by using mice with lymphatic endothelial cellspecific deficiency of VEGFR2 was compromised by the complication that loss of VEGFR2 abolishes VEGFR3 expression in these mice (67). Nevertheless, given that VEGFR2 and VEGFR3 can form heterodimers to transduce the VEGF-C or VEGF-D signal, a role for VEGFR2 in regulating lymphangiogenesis is plausible. To definitively assess the role of VEGFR2 in modulating diabetic lymphangiogenesis, we performed a tail edema study in LEC-iDKOFlt $4^{\text {eGFP/+ }} /$ STZ/HFD mice, in which VEGFR3 levels are reduced without affecting VEGFR2 levels. These mice exhibited impaired tail edema resolution compared with LEC-iDKO/STZ/HFD mice, suggesting a rather minor role for VEGFR2 in regulating lymphangiogenesis in diabetic conditions (Supplemental Figure 14, $\mathrm{A}$ and $\mathrm{B})$. Furthermore, our previous unpublished data revealed that reducing VEGFR2 levels by genetically removing one allele of VEGFR2 did not impact the enhanced VEGFR3 levels and subsequent phenotypic changes in lymphatic development observed in epsin mutant mice, indicating that VEGFR3 plays a dominant role during lymphatic development and regeneration, especially in the mouse models that we are currently utilizing.

As expected, we observed that the abundance of VEGFR3 at the cell surface is controlled by VEGF-C-induced endocytosis and degradation in physiological conditions. However, under diabetic conditions, the abundance of cell-surface VEGFR3 is regulated not only by VEGF-C-induced endocytosis and degradation but also by ROS-mediated phosphorylation and subsequent degradation. The balance between pro- and antilymphangiogenic force plays a key role in lymphatic regeneration after injury or under pathological conditions such as obesity and diabetes (68). Here, we believe we are the first to demonstrate that in WT/STZ/HFD diabetic mice, VEGFR3 is degraded by a VEGF-C/VEGFR3/epsindependent pathway in addition to ROS-mediated phosphorylation and epsin-guided degradation. However, epsin deficiency impairs both VEGF-C-induced VEGFR3 and ROS-triggered VEGFR3 degradation, thus safeguarding the abundance of VEGFR3 to sustain appropriate lymphangiogenesis in LEC-iDKO/STZ/HFD mice. Given the importance of VEGFR3 in regeneration of tissue microcirculation, the intrinsic ROS-mediated degradative pathways mediating the loss of VEGFR3 and the precise downstream regulatory machinery of VEGFR3 degradation in diabetes mellitus warrant further investigation.

In models of corneal inflammation, lymphatic vessels arise de novo within the corneal stroma at a distance from the limbus, a pathological phenomenon which is generally considered harmful $(69,70)$. However, our corneal lymphangiogenesis model showed that the nascent lymphatic vessels sprouted from the limbus, not arising de novo within the corneal stroma, indicating that our corneal micropocket assay differs from previously performed corneal inflammation models (69-71). Importantly, we did not observe macrophage infiltration in the Matrigel plug assay in our STZ/HFD diabetic models. Additionally, mice lacking STZ treatment but fed a HFD for 12 weeks showed no significant change in VEGFR3 in both the mRNA and protein levels in LECs (data not shown), suggesting that our STZ/HFD diabetic models are different from the chronic inflammatory obesity model and the diabetic model with longterm HFD feeding (72). Furthermore, our tail edema model assay showed that deficiency of epsins restored insufficient lymphangiogenesis in vivo and improved the resolution of tail edema by preventing the degradation of VEGFR3 in diabetes. This is consistent with previous studies demonstrating that insufficient lymphangiogenesis induces lymphedema $(39,61)$. Although both VEGF-C and VEGF-D can bind VEGFR3, the VEGF-C/VEGFR3 signaling axis 
is the most well-studied pathway for lymphangiogenesis $(2,3,20$, 22-24). As expected, by using both VEGF-C152S (a mutant form of VEGF-C that specifically binds to VEGFR3 but not VEGFR2) and the $\triangle \mathrm{N} \triangle \mathrm{C}$ form (a high-affinity form of VEGF-C without the $\mathrm{N}$ - and C-terminal domains), we observed growth of lymphatic vessels but not blood vessels stimulated by exogenous VEGF-C in our corneal micropocket assay (Figure 1B). Furthermore, in the tail edema analysis, VEGF-C levels were not dramatically changed in either the serum or tail edema tissues from WT, LEC-iDKO, WT/ STZ/HFD, and LEC-iDKO/STZ/HFD mice (Supplemental Figure 11 ), suggesting that hyperglycemia-induced oxidative stress intrinsically targets VEGFR3 for degradation independent of VEGF-C stimulation, and loss of epsins can rescue lymphangiogenesis by prohibiting the detrimental degradation of VEGFR3 in diabetes. Given that we observed minimal alteration of VEGF-C levels among 4 groups of mice in our experiments, we postulate that levels of VEGF-D would be similar as well in all groups of mice; however, experimental evidence remains to be gathered.

Our findings in ChIP and luciferase assays demonstrated that diabetic oxidative stress upregulated expression of epsins through c-Src-mediated and AP-1-driven transcriptional regulation. Bioinformatic studies suggest that FOXC2 could potentially regulate expression of epsins, and previous studies indicate that FOXC2 plays important roles in developing lymphatics as well as in lymphatic valve maintenance $(2,73,74)$. We found that FOXC2 expression was markedly reduced in STZ/HFD diabetic models compared with the control group, suggesting that upregulation of epsin in diabetes is unlikely driven by FOXC2 in our mouse models (data not shown). Nonetheless, whether FOXC2 mediates the expression of other prolymphangiogenic molecules that could be beneficial to lymphangiogenesis in type 2 diabetes remains to be studied.

Diabetic patients with uncontrolled hyperglycemia often suffer from consequences of severely delayed wound healing and chronic secondary lymphedema partly due to impaired angiogenesis and lymphangiogenesis $(12,13)$. Mechanisms by which diabetes impairs wound healing and lymphedema resolution remain unclear. We believe we are the first to report that high-glucoseinduced oxidative stress also causes the intrinsic phosphorylation of VEGFR3 at the Golgi and subsequent trafficking to the lysosome for degradation. Loss of epsins restored VEGFR3 signaling and lymphangiogenesis in LECs exposed to hyperglycemia, improved corneal lymphatic growth, and rescued secondary tail edema in diabetic mice. Our findings complement previous studies in which the stimulation of lymphangiogenesis via the VEGFR3 pathway inhibited chronic skin inflammation $(23,63)$. Lastly, our findings expose therapeutic potential for targeted inhibition of epsins within the lymphatic endothelium, particularly resolution of lymphedema owing to hypoplastic lymphangiogenesis and the recovery of wound healing in uncontrolled hyperglycemic diabetic patients.

\section{Methods}

Animal models. Prox1-Cre-ER ${ }^{T 2}$ mice were a gift from R.S. Srinivasan (OMRF, Oklahoma City, Oklahoma) and have been previously

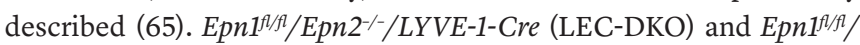
Epn2-/-Prox1-Cre-ER $R^{T 2}$ (LEC-iDKO) mice were generated by crossing Epn1 $1^{f l f l} / E p n 2^{-/-}$mice with LYVE-1-Cre mice or Prox1-Cre-ER ${ }^{T 2}$ mice as previously described (32-34). Adult Epn1 $1^{t / f l} / E p n 2^{-/-} /$Prox1-Cre-ER ${ }^{T 2}$ mice on an epsin 2-null background were selectively ablated of epsin 1 in LECs by administering 4-hydroxytamoxifen at 8 to 10 weeks of age. Epn $1^{f / f l} / E p n 2^{-/-}$littermate mice without Prox1-Cre-ER $R^{T 2}$ or WT mice with Prox1-Cre-ER ${ }^{T 2}$ were utilized as controls and denoted as WT. The mice heterozygous for VEGFR3 on an inducible LEC-specific epsins 1 and 2 deletion background (LEC-iDKO-Flt4 ${ }^{\mathrm{eGFP} /+}$ ) were generated by crossing the LEC-iDKO mice with VEGFR3-eGFP knockin mice $\left(F l t 4^{\mathrm{GGFP} /+}\right)$ as previously described $(34,55)$. The leptin-deficient diabetic $d b / d b$ mice (JAX mice) were purchased from Charles River. Additional methods are provided in Supplemental Methods.

Study approval. All animal studies were performed in compliance with institutional guidelines and were approved by the IACUC, Boston Children's Hospital at Harvard Medical School, Boston, MA. Studies using human samples were carried out in accordance with approved guidelines from the human research ethics committee of the Second Xiagya Hospital, Central South University, China.

Statistics. Data are mean \pm SEM. GraphPad Prism 7 or SPSS software (IBM) was used to perform the statistical analysis. Data were analyzed by 2-tailed Student's $t$ test. One-way ANOVA, 2-way ANOVA, and 3-way ANOVA followed by Tukey's post hoc analysis were used for multiple comparisons where appropriate. $P<0.05$ was considered significant.

\section{Author contributions}

HW and HC designed, analyzed, and interpreted the data. HW conducted most in vitro and in vivo experiments and handled the data presentation. HNAR, XL, and SP performed signaling experiments in vitro. YD performed the eNOS effects and serum VEGF-C measurement. YL, AW, KHTT, MRM, and XC conducted lymphatic vessel imaging and helped with immunostaining. WY and LX conducted the human study. AEB and LB conducted the mouse micropocket assay. MLB, HNAR, and KS performed LEC cell proliferation, migration, and tube formation experiments in vitro. BC helped with the luciferase assay. SW and SK performed mouse breeding and genotyping, and provided technical support. SW and YL helped with statistical analysis. RJD, RSS, JBD, DRB, JB, and JX helped with animal models and discussions. HW and HC wrote the manuscript.

\section{Acknowledgments}

We thank Marsha Moses and Jing Chen for helpful discussions and critical reading of our manuscript, Harry Leung for help with the multiple-proton microscopy imaging, Avner Adini and Hiroko Kishikawa for assistance with flow cytometry, Matthew Cribb for assistance with the NIR imaging system, and Haojie $\mathrm{Fu}$ for assistance with isolation of mouse corneas. This work was supported in part by NIH grants R01HL-093242, R01HL-118676, R01HL-137229, and the Established Investigator Award from the American Heart Association (AHA) to HC; NIH grant R01HL-133216 to HC, RSS, and JBD; NIH grant R01HL-130845 to HC and JX; NIH (P20GM104934), American Diabetes Association (ADA, 1-12-JF-58), AHA Scientist Development Grant (SDG, 10SDG, 14BGIA20030027), and Oklahoma Center for the Advancement of Science and Technology (OCAST, HR11-200, HR14-062, and HR17-046) grants to JX; NIH grant R01HL-096384-07 to JB; AHA SDG grant 17SDG33410868 to HW; AHA SDG grant 17SDG33630161 to KS; OCAST grants AR11- 
043, HR14-056, and 12SDG8760002 to YD; AHA fellowship 16POST31210039 to HNAR; AHA grant 15PRE21400010 and NIH grant 1F31HL127982-01 to MLB; National Foundation Committee of Natural Sciences of China grant NSFC 81570658 to XL.
Address correspondence to: Hong Chen, Vascular Biology Program, Harvard Medical School, Boston Children's Hospital, Boston, Massachusetts 02115, USA. Phone: 617.919.6304; Email: hong.chen@childrens.harvard.edu.
1. Alitalo K, Tammela T, Petrova TV. Lymphangiogenesis in development and human disease. Nature. 2005;438(7070):946-953.

2. Tammela T, Alitalo K. Lymphangiogenesis: molecular mechanisms and future promise. Cell. 2010;140(4):460-476.

3. Aspelund A, Robciuc MR, Karaman S, Makinen T, Alitalo K. Lymphatic system in cardiovascular medicine. Circ Res. 2016;118(3):515-530.

4. Maruyama K, Asai J, Ii M, Thorne T, Losordo DW, D’Amore PA. Decreased macrophage number and activation lead to reduced lymphatic vessel formation and contribute to impaired diabetic wound healing. Am J Pathol. 2007;170(4):1178-1191.

5. Saaristo A, et al. Vascular endothelial growth factor- $\mathrm{C}$ accelerates diabetic wound healing. Am J Pathol. 2006;169(3):1080-1087.

6. Paavonen K, Puolakkainen P, Jussila L, Jahkola T, Alitalo K. Vascular endothelial growth factor receptor-3 in lymphangiogenesis in wound healing. Am J Pathol. 2000;156(5):1499-1504.

7. Scallan JP, Hill MA, Davis MJ. Lymphatic vascular integrity is disrupted in type 2 diabetes due to impaired nitric oxide signalling. Cardiovasc Res. 2015;107(1):89-97.

8. Asai J, et al. Topical simvastatin accelerates wound healing in diabetes by enhancing angiogenesis and lymphangiogenesis. Am J Pathol. 2012;181(6):2217-2224.

9. Forbes JM, Cooper ME. Mechanisms of diabetic complications. Physiol Rev. 2013;93(1):137-188.

10. Xu J, Zou MH. Molecular insights and therapeutic targets for diabetic endothelial dysfunction. Circulation. 2009;120(13):1266-1286.

11. Rutkowski JM, Moya M, Johannes J, Goldman $J$, Swartz MA. Secondary lymphedema in the mouse tail: lymphatic hyperplasia, VEGF-C upregulation, and the protective role of MMP-9. Microvasc Res. 2006;72(3):161-171.

12. Jeffcoate WJ, Harding KG. Diabetic foot ulcers. Lancet. 2003;361(9368):1545-1551.

13. Lin CT, Ou KW, Chang SC. Diabetic foot ulcers combination with lower limb lymphedema treated by staged charles procedure: case report and literature review. PakJMed Sci.2013;29(4):1062-1064.

14. $\mathrm{Cho} \mathrm{CH}$, et al. $\mathrm{COMP}$-angiopoietin-1 promotes wound healing through enhanced angiogenesis, lymphangiogenesis, and blood flow in a diabetic mouse model. Proc Natl Acad Sci U S A. 2006;103(13):4946-4951.

15. Gousopoulos E, et al. An important role of VEGF$\mathrm{C}$ in promoting lymphedema development. JInvest Dermatol. 2017;137(9):1995-2004.

16. Kukk E, et al. VEGF-C receptor binding and pattern of expression with VEGFR-3 suggests a role in lymphatic vascular development. Development. 1996;122(12):3829-3837.

17. Goldman J, Le TX, Skobe M, Swartz MA. Overexpression of VEGF-C causes transient lymphatic hyperplasia but not increased lymphangiogenesis in regenerating skin. Circ Res.
2005;96(11):1193-1199.

18. Kaipainen A, et al. Expression of the fms-like tyrosine kinase 4 gene becomes restricted to lymphatic endothelium during development. Proc Natl Acad Sci USA. 1995;92(8):3566-3570.

19. Dumont DJ, et al. Cardiovascular failure in mouse embryos deficient in VEGF receptor-3. Science. 1998;282(5390):946-949.

20. Zhang L, et al. VEGFR-3 ligand-binding and kinase activity are required for lymphangiogenesis but not for angiogenesis. Cell Res. 2010;20(12):1319-1331.

21. Karkkainen MJ, et al. Vascular endothelial growth factor $\mathrm{C}$ is required for sprouting of the first lymphatic vessels from embryonic veins. Nat Immunol. 2004;5(1):74-80.

22. Tammela T, et al. Therapeutic differentiation and maturation of lymphatic vessels after lymph node dissection and transplantation. Nat Med. 2007;13(12):1458-1466.

23. Hagura A, Asai J, Maruyama K, Takenaka H, Kinoshita S, Katoh N. The VEGF-C/VEGFR3 signaling pathway contributes to resolving chronic skin inflammation by activating lymphatic vessel function. J Dermatol Sci. 2014;73(2):135-141.

24. Jeltsch M, et al. Hyperplasia of lymphatic vessels in VEGF-C transgenic mice. Science. 1997;276(5317):1423-1425.

25. Kriehuber E, et al. Isolation and characterization of dermal lymphatic and blood endothelial cells reveal stable and functionally specialized cell lineages. JExp Med. 2001;194(6):797-808.

26. Deng Y, Zhang X, Simons M. Molecular controls of lymphatic VEGFR3 signaling. Arterioscler Thromb Vasc Biol. 2015;35(2):421-429.

27. Mäkinen T, et al. Isolated lymphatic endothelial cells transduce growth, survival and migratory signals via the VEGF-C/D receptor VEGFR-3. EMBO J. 2001;20(17):4762-4773.

28. Warren CM, Ziyad S, Briot A, Der A, Iruela-Arispe ML. A ligand-independent VEGFR2 signaling pathway limits angiogenic responses in diabetes. Sci Signal. 2014;7(307):ra1.

29. Chen C, Zhuang X. Epsin 1 is a cargo-specific adaptor for the clathrin-mediated endocytosis of the influenza virus. Proc Natl Acad Sci U S A. 2008;105(33):11790-11795.

30. Chen $\mathrm{H}$, et al. Epsin is an EH-domain-binding protein implicated in clathrin-mediated endocytosis. Nature. 1998;394(6695):793-797.

31. Chen H, De Camilli P. The association of epsin with ubiquitinated cargo along the endocytic pathway is negatively regulated by its interaction with clathrin. Proc Natl Acad Sci US A. 2005;102(8):2766-2771.

32. Chen $\mathrm{H}$, et al. Embryonic arrest at midgestation and disruption of Notch signaling produced by the absence of both epsin 1 and epsin 2 in mice. Proc Natl Acad Sci U S A. 2009;106(33):13838-13843.

33. Pasula S, et al. Endothelial epsin deficiency decreases tumor growth by enhancing VEGF sig- naling. J Clin Invest. 2012;122(12):4424-4438.

34. Liu X, et al. Temporal and spatial regulation of epsin abundance and VEGFR3 signaling are required for lymphatic valve formation and function. Sci Signal. 2014;7(347):ra97.

35. Rahman HNA, et al. Selective targeting of a novel epsin-VEGFR2 interaction promotes VEGF-mediated angiogenesis. Circ Res. 2016;118(6):957-969.

36. Shin WS, Rockson SG. Animal models for the molecular and mechanistic study of lymphatic biology and disease. Ann N Y Acad Sci. 2008;1131:50-74.

37. Ly CL, Kataru RP, Mehrara BJ. Inflammatory manifestations of lymphedema. Int J Mol Sci. 2017;18(1):171.

38. Moriguchi P, Sannomiya P, Lara PF, Oliveira-Filho RM, Greco KV, Sudo-Hayashi LS. Lymphatic system changes in diabetes mellitus: role of insulin and hyperglycemia. Diabetes Metab Res Rev. 2005;21(2):150-157.

39. Alitalo K. The lymphatic vasculature in disease. Nat Med. 2011;17(11):1371-1380.

40. Yin $\mathrm{N}$, et al. Lymphangiogenesis is required for pancreatic islet inflammation and diabetes. PLoS One. 2011;6(11):e28023.

41. Nakao S, Hafezi-Moghadam A, Ishibashi T. Lymphatics and lymphangiogenesis in the eye. JOphthalmol. 2012;2012:783163.

42. Ling S, et al. Development of new lymphatic vessels in alkali-burned corneas. Acta Ophthalmol. 2009;87(3):315-322.

43. Cursiefen C, et al. VEGF-A stimulates lymphangiogenesis and hemangiogenesis in inflammatory neovascularization via macrophage recruitment. J Clin Invest. 2004;113(7):1040-1050.

44. Rogers MS, Birsner AE, D'Amato RJ. The mouse cornea micropocket angiogenesis assay. Nat Protoc. 2007;2(10):2545-2550.

45. Cao R, et al. Mouse corneal lymphangiogenesis model. Nat Protoc. 2011;6(6):817-826.

46. Yamaguchi $\mathrm{T}$, et al. Interspecies organogenesis generates autologous functional islets. Nature. 2017;542(7640):191-196.

47. King AJ. The use of animal models in diabetes research. Br J Pharmacol. 2012;166(3):877-894.

48. Gilbert ER, Fu Z, Liu D. Development of a nongenetic mouse model of type 2 diabetes. Exp Diabetes Res. 2011;2011:416254.

49. Reuter S, Gupta SC, Chaturvedi MM, Aggarwal BB. Oxidative stress, inflammation, and cancer: how are they linked? Free Radic Biol Med. 2010;49(11):1603-1616.

50. Rivat C, et al. Synergistic cooperation between the AP-1 and LEF-1 transcription factors in activation of the matrilysin promoter by the src oncogene: implications in cellular invasion. FASEB J. 2003;17(12):1721-1723.

51. Robertson RP, Harmon J, Tran PO, Poitout V. Beta-cell glucose toxicity, lipotoxicity, and chronic oxidative stress in type 2 diabetes. 
Diabetes. 2004;53 Suppl 1:S119-S124.

52. Wang JF, Zhang X, Groopman JE. Activation of vascular endothelial growth factor receptor- 3 and its downstream signaling promote cell survival under oxidative stress. J Biol Chem. 2004;279(26):27088-27097.

53. Rutkowski JM, Boardman KC, Swartz MA. Characterization of lymphangiogenesis in a model of adult skin regeneration. Am J Physiol Heart Circ Physiol. 2006;291(3):H1402-H1410.

54. Piller NB. Macrophage and tissue changes in the developmental phases of secondary lymphoedema and during conservative therapy with benzopyrone. Arch Histol Cytol. 1990;53 Suppl:209-218.

55. Ichise T, Yoshida N, Ichise H. H-, N- and Kras cooperatively regulate lymphatic vessel growth by modulating VEGFR3 expression in lymphatic endothelial cells in mice. Development. 2010;137(6):1003-1013.

56. Podgrabinska S, Braun P, Velasco P, Kloos B, Pepper MS, Skobe M. Molecular characterization of lymphatic endothelial cells. Proc Natl Acad Sci U S A. 2002;99(25):16069-16074.

57. Zheng W, Aspelund A, Alitalo K. Lymphangiogenic factors, mechanisms, and applications. JClin Invest. 2014;124(3):878-887.

58. Ji RC. Characteristics of lymphatic endothelial cells in physiological and pathological condi- tions. Histol Histopathol. 2005;20(1):155-175.

59. Kaufmann A, Molnár B, Craciun C, Itcus A. Diabetic lymphangiopathy. An optical and electron microscopic study. Lymphology. 1980;13(4):202-206.

60. Escobedo N, et al. Restoration of lymphatic function rescues obesity in Prox1-haploinsufficient mice. JCI Insight. 2016;1(2):e85096.

61. Kim H, Kataru RP, Koh GY. Regulation and implications of inflammatory lymphangiogenesis. Trends Immunol. 2012;33(7):350-356.

62. Haemmerle M, et al. Enhanced lymph vessel density, remodeling, and inflammation are reflected by gene expression signatures in dermal lymphatic endothelial cells in type 2 diabetes. Diabetes. 2013;62(7):2509-2529.

63. Huggenberger R, Ullmann S, Proulx ST, Pytowski B, Alitalo K, Detmar M. Stimulation of lymphangiogenesis via VEGFR-3 inhibits chronic skin inflammation. JExp Med.2010;207(10):2255-2269.

64. Bianchi A, Painter KJ, Sherratt JA. A mathematical model for lymphangiogenesis in normal and diabetic wounds. J Theor Biol. 2015;383:61-86.

65. Srinivasan RS, et al. Lineage tracing demonstrates the venous origin of the mammalian lymphatic vasculature. Genes Dev. 2007;21(19):2422-2432.

66. Dong $\mathrm{Y}$, et al. Motif mimetic of epsin perturbs tumor growth and metastasis. JClin Invest. 2015;125(12):4349-4364.

67. Benedito R, et al. Notch-dependent VEGFR3 upreg- ulation allows angiogenesis without VEGF-VEGFR2 signalling. Nature. 2012;484(7392):110-114.

68. Gardenier JC, et al. Topical tacrolimus for the treatment of secondary lymphedema. Nat Commun. 2017;8:14345.

69. Maruyama K, et al. Inflammation-induced lymphangiogenesis in the cornea arises from CD11b-positive macrophages. JClin Invest. 2005;115(9):2363-2372.

70. Hos D, et al. IL-10 indirectly regulates corneal lymphangiogenesis and resolution of inflammation via macrophages. Am J Pathol. 2016;186(1):159-171.

71. Dietrich T, et al. Cutting edge: lymphatic vessels, not blood vessels, primarily mediate immune rejections after transplantation. JImmunol. 2010;184(2):535-539.

72. Calligaris SD, et al. Mice long-term high-fat diet feeding recapitulates human cardiovascular alterations: an animal model to study the early phases of diabetic cardiomyopathy. PLOS ONE. 2013;8(4):e60931.

73. Sabine A, et al. FOXC2 and fluid shear stress stabilize postnatal lymphatic vasculature. JClin Invest. 2015;125(10):3861-3877.

74. Fatima A, et al. Foxc1 and Foxc2 deletion causes abnormal lymphangiogenesis and correlates with ERK hyperactivation. J Clin Invest. 2016;126(7):2437-2451. 